JOURNAL OF OPTIMIZATION, DIFFERENTIAL EQUATIONS AND THEIR APPLICATIONS (JODEA) Volume 27, Issue 2, December 2019, pp. 60-95, DOI 10.15421/141908

ISSN (print) 2617-0108

ISSN (on-line) 2663-6824

\title{
ON OPTIMAL 2-D DOMAIN SEGMENTATION PROBLEM VIA PIECEWISE SMOOTH APPROXIMATION OF A SELECTIVE TARGET MAPPING
}

\author{
Volodymyr V. Hnatushenko, Peter I. Kogut $\dagger^{\dagger}$ Mykola V. Uvarov ${ }^{\ddagger}$
}

\begin{abstract}
In this paper we propose a new technique for the solution of the image segmentation problem which is based on the concept of a piecewise smooth approximation of some target functional. We discuss in details the consistency of the new statement of segmentation problem and its solvability. We focus our main intension on the rigor mathematical substantiation of the proposed approach, deriving the corresponding optimality conditions, and show that the new optimization problem is rather flexible and powerful model to the study of variational image segmentation problems. We illustrate the accuracy and efficiency of the proposed algorithm by numerical experiences.
\end{abstract}

Key words: Optimal segmentation problem, piecewise constant approximation, optimality conditions, variational problem..

2010 Mathematics Subject Classification: 49Q20, 49K10, 49J45, 26B30.

Communicated by Prof. P. O. Kasyanov

\section{Introduction}

In this paper we discuss a new coupled variational problem which is suggested by applications to satellite image segmentation. From practical point of view, image segmentation is the process of dividing an image into several areas with features and extracting the target of interest. in particular, in agricultural crop field classification, one of a fundamental problem is to provide a disjunctive decomposition of a fixed domain $\Omega \subset \mathbb{R}^{2}$ onto finite number of nonempty subsets $\Omega=\Omega_{1} \cup \Omega_{2} \cup \cdots \cup \Omega_{K}$ such that each of these subsets could be associated with a crop that is grown in this area, or with a forest regions, or water zones, and so on, and this correspondence must be established at rather high level of accuracy. Therefore, one important premise of object segmentation is to construct image objects that have homogeneous features $[20,25,30]$. In the agricultural applications, we consider the IPVI-characteristic as the main feature of such images. At the same time, a precise consideration of this problem demonstrates that the quantitative interpretation of remote sensing information from vegetation is a complex

\footnotetext{
* Department of Information Systems and Technologies, Dnipro University of Technology, av. Dmytra Yavornytskoho, 19, 49005, Dnipro, Ukraine; EOS Data Analytics Ukraine, Gagarin av., 103a, Dnipro, Ukraine, vladimir.gnatushenko@eosda.com

${ }^{\dagger}$ Department of Differential Equations, Oles Honchar Dnipro National University, 72, Gagarin av., Dnipro, 49010, Ukraine; EOS Data Analytics Ukraine, Gagarin av., 103a, Dnipro, Ukraine p.kogut@i.ua, peter.kogut@eosda.com

${ }^{\ddagger}$ EOS Data Analytics Ukraine, Desyatynny lane, 5, 01001 Kyiv, Ukraine, nikolay.uvarov@eosda.com (C) V. V. Hnatushenko, P. I. Kogut, M. V. Uvarov, 2019.
} 
task. Many studies have limited this interpretation by assumption that the extracting vegetation information uniformly and smoothly distributed within the particular crop fields. However, this assumption is evidently broken when trying to apply these type vegetation indices on heterogeneous canopies such as plantations with a mixed combination of soil, weeds, and other crops, or plantation where the vegetation of interest has different IPVI-characteristic due to spacial variability.

The main idea, we try to realize in the new setting of variational problem, can be briefly described as follows. At the first step, we consider the following collection of variational problems

$$
\begin{aligned}
& J_{i}(u) \longrightarrow \inf , \quad u \in \Xi \subset B V(\Omega), \\
& J_{i}(u)=\int_{\Omega} d|D u|+\lambda_{1}\left\|u-u_{i, d}\right\|_{H^{-1}(\Omega)}^{2}+\lambda_{2}\left\|B_{i}(u)-v_{i, d}\right\|_{L^{2}(\Omega)}^{2},
\end{aligned}
$$

where $B_{i}: L^{2}(\Omega) \rightarrow L^{2}(\Omega)$ is a linear continuous operator.

We show that each of the constrained minimization problems (1.1)-(1.2) is consistent and admits a unique solution $u_{i}^{\text {opt }}$ for each $i=1, \ldots, M$. Further we pass from the solutions $\left[u_{1}^{o p t}, u_{2}^{o p t}, \ldots, u_{M}^{o p t}\right]^{T}$ of the problems (1.1)-(1.2) to their 'locally smoothed' versions. Formally we describe this passage by introduction of some nonlinear sequentially continuous operators $V_{i}: B V(\Omega) \rightarrow B V(\Omega)$, i.e.

$$
\left[u_{1}^{o p t}, u_{2}^{o p t}, \ldots, u_{M}^{o p t}\right] \Longrightarrow\left[V_{1}\left(u_{1}^{o p t}\right), V_{2}\left(u_{2}^{o p t}\right), \ldots, V_{M}\left(u_{M}^{o p t}\right)\right] .
$$

As a final step, we introduce a special target mapping

$$
V:[B V(\Omega)]^{M} \rightarrow L^{\infty}(\Omega)
$$

such that

$$
V\left(u_{1}, \ldots, u_{M}\right)=V\left(V_{i}\left(u_{1}\right), \ldots, V_{i}\left(u_{M}\right)\right), \quad \forall\left(u_{1}, \ldots, u_{M}\right) \in[B V(\Omega)]^{M}
$$

and consider the problem of a piecewise smooth approximation of the distribution $V\left(\mathbf{u}^{o p t}\right)=V\left(u_{1}^{o p t}\right), \ldots, V_{i}\left(u_{M}^{o p t}\right)$ in domain $\Omega$. Loosely speaking, this problem consists in finding a two-phase decomposition $\Omega=E_{1} \cup E_{2}$ such that the distribution $V\left(\mathbf{u}^{\text {opt }}\right) \in L^{\infty}(\Omega)$ varies slowly with respect to the $L^{r}\left(E_{i}\right)$-norm within each subset $E_{i}$, and the distribution $V\left(\mathbf{u}^{o p t}\right) \in L^{\infty}(\Omega)$ varies rapidly (or discontinuously) across most of the boundary between $E_{1}$ and $E_{2}$.

It is worth to note that minimization problems like (1.1)-(1.2) with an $\mathrm{H}^{-1}$ constraint found growing interest in recent years due to several advantages to the $L^{2}$-constrained problems. In particular, the benefit to involve $H^{-1}$-norm in image processing models is well-know (see [6] for a general overview on this topic). From practical point of view, the $H^{-1}(\Omega)$ space is intrinsically more appropriate for the modeling texture or oscillatory pattern and, in fact, it provides the norm 
which is smaller than the $L^{2}$-norm. As for the nonlinear sequentially continuous operators $V_{i}: B V(\Omega) \rightarrow B V(\Omega)$ that have been mentioned before, they can be chosen in a different way. However, for practical implementation of this step, we consider the mappings $u \mapsto V_{i}(u)$ as the resolvent operator of Alvarez, Lions, and Morel model [2] that takes the form of some initial-boundary value problem for parabolic equation with a nonlinear anisotropic diffusion operator in its principle part and with the function $u$ in its initial condition.

The paper is organized as follows. In Section 2 we give some preliminaries related with the space of functions of bounded variation. The precise setting of coupled optimization problem and its previous analysis is given in Section 3. In particular, we define the concept of feasible solutions to that problem and show that, under some proper assumptions, this problem has a solution. The aim of Section 4 is to derive optimality conditions and provide their substantiation. In Section 5 we discuss the practical implementation of the considered problem to the satellite image processing related to the monitoring of crop fields. We show that this problem can be considered as a particular case of the proposed couple optimization problem. We also give the results of numerical simulation with the real-life satellite images which illustrate the accuracy and efficiency of the proposed algorithm.

\section{Auxiliaries}

Let $\Omega$ be a bounded open subset of $\mathbb{R}^{2}$ with a Lipschitz boundary. For any subset $E \subset \Omega$ we denote by $|E|$ its 2 -dimensional Lebesgue measure $\mathcal{L}^{2}(E)$. For a subset $E \subseteq \Omega$ let $\bar{E}$ denote its closure and $\partial E$ its boundary. We define the characteristic function $\chi_{E}$ of $E$ by

$$
\chi_{E}(x):= \begin{cases}1, & \text { for } x \in E, \\ 0, & \text { otherwise. }\end{cases}
$$

For a function $u$ we denote by $\left.u\right|_{E}$ its restriction to the set $E \subseteq \Omega$, and by $u^{\partial E}$ its trace on $\partial E$. Let $C_{0}^{\infty}(\Omega)$ be the infinitely differentiable functions with compact support in $\Omega$. The $k$-dimensional Hausdorff measure is denoted by $\mathcal{H}^{k}$, and $\mu\llcorner E$ is the restriction of a measure $\mu$ to the set $E$. For a Banach space $X$ its dual is $X^{*}$ and $\langle\cdot, \cdot\rangle_{X^{*}, X}$ is the duality form on $X^{*} \times X$. By $\rightarrow$ and $\stackrel{*}{\rightarrow}$ we denote the weak and weak ${ }^{*}$ convergence in normed spaces.

Throughout the paper we will often use the concept of weak and strong convergence in $L^{1}(\Omega)$. Let $\left\{f_{n}\right\}_{n \in \mathbb{N}}$ be a bounded sequence of functions in $L^{1}(\Omega)$. We recall that $\left\{f_{n}\right\}_{n \in \mathbb{N}}$ is called equi-integrable on $\Omega$, if for any $\delta>0$ there is a $\tau=\tau(\delta)$ such that $\int_{S}\left|f_{n}\right| d x<\delta$ for every measurable subset $S \subset \Omega$ of Lebesgue measure $|S|<\tau$. Then the following assertions are equivalent for $L^{1}(\Omega)$-bounded sequences (see, for instance, $[5,16]$ ):

(i) a sequence $\left\{f_{k}\right\}_{k \in \mathbb{N}}$ is weakly convergent in $L^{1}(\Omega)$;

(ii) the sequence $\left\{f_{k}\right\}_{k \in \mathbb{N}}$ is equi-integrable. 
Theorem 2.1 ( [5]). If a bounded sequence $\left\{f_{k}\right\}_{k \in \mathbb{N}} \subset L^{1}(\Omega)$ is equi-integrable and $f_{k} \rightarrow f$ almost everywhere in $\Omega$, then $f_{k} \rightarrow f$ strongly in $L^{1}(\Omega)$.

We remind here the most common definitions of some functional spaces that we will use later on.

\subsection{The Space $H^{-1}(\Omega)$}

Let $\mathcal{D}^{\prime}(\Omega)$ be the dual of the space $C_{0}^{\infty}(\Omega)$, i.e. $\mathcal{D}^{\prime}(\Omega)$ is the space of distributions in $\Omega$. By $H_{0}^{1}(\Omega)$ we denote the closure of $C_{0}^{\infty}(\Omega)$-functions with respect to the norm

$$
\|u\|=\left[\|u\|_{L^{2}(\Omega)}^{2}+\|\nabla u\|_{L^{2}\left(\Omega ; \mathbb{R}^{2}\right)}^{2}\right]^{\frac{1}{2}}=\left(\int_{\Omega}\left[u^{2}(x)+|\nabla u(x)|_{\mathbb{R}^{2}}^{2}\right] d x\right)^{\frac{1}{2}} .
$$

Then $H_{0}^{1}(\Omega)$ is a Banach space and the norm in $H_{0}^{1}(\Omega)$ can be defined by

$$
\|y\|_{H_{0}^{1}(\Omega)}=\left(\int_{\Omega}\|\nabla y\|_{\mathbb{R}^{N}}^{2} d x\right)^{1 / 2} .
$$

We denote the dual of $H_{0}^{1}(\Omega)$ by $H^{-1}(\Omega)$. Then (see [26, p.401]), $H^{-1}(\Omega)$ is isometrically isomorphic to the Hilbert space of all distributions $F \in \mathcal{D}^{\prime}(\Omega)$ satisfying

$$
F=g_{0}+\sum_{i=1}^{2} \frac{\partial g_{i}}{\partial x_{i}} \quad \text { for some } g_{0}, g_{1}, g_{2} \in L^{2}(\Omega)
$$

with

$$
\|F\|_{H^{-1}(\Omega)}=\inf \left\{\left(\sum_{i=0}^{2}\left\|g_{i}\right\|_{L^{2}(\Omega)}^{2}\right)^{1 / 2}: F=g_{0}+\sum_{i=1}^{2} \frac{\partial g_{i}}{\partial x_{i}}\right\} .
$$

Let's fix an arbitrary element $u^{*}$ of $H^{-1}(\Omega)$. Then there exists a vectorfunction $F=\left[F_{1}, F_{2}\right]$ in $L^{2}\left(\Omega ; \mathbb{R}^{2}\right)$ such that

$$
\begin{aligned}
\left\langle u^{*}, u\right\rangle_{H^{-1}(\Omega) ; H_{0}^{1}(\Omega)} & =\int_{\Omega}(F, \nabla u)_{\mathbb{R}^{2}} d x \\
& =\int_{\Omega}\left[F_{1} \frac{\partial u}{\partial x_{1}}+F_{2} \frac{\partial u}{\partial x_{2}}\right] d x, \quad \forall u \in H_{0}^{1}(\Omega) .
\end{aligned}
$$

Therefore, it is clear now that

$$
\left\|u^{*}\right\|_{H^{-1}(\Omega)} \leqslant \sqrt{\int_{\Omega}\left(F_{1}^{2}(x)+F_{2}^{2}(x)\right) d x} .
$$

On the other hand, due to the Lax-Milgram Lemma, the Dirichlet boundary value problem

$$
-\Delta y=u^{*} \text { in } \Omega, \quad y=0 \text { on } \partial \Omega,
$$


has a unique solution $y=(-\Delta)^{-1} u^{*} \in H_{0}^{1}(\Omega)$ for each $u^{*} \in H^{-1}(\Omega)$, where

$$
\Delta=\frac{\partial^{2}}{\partial x_{1}^{2}}+\frac{\partial^{2}}{\partial x_{2}^{2}}: H_{0}^{1}(\Omega) \rightarrow H^{-1}(\Omega)
$$

stands for the Laplace operator. Moreover, in view of the energy equality

$$
\int_{\Omega}(\nabla y, \nabla y)_{\mathbb{R}^{2}} d x=\|\nabla y\|_{L^{2}\left(\Omega ; \mathbb{R}^{2}\right)}^{2}=\|y\|_{H_{0}^{1}(\Omega)}^{2}=\left\langle u^{*}, y\right\rangle_{H^{-1}(\Omega) ; H_{0}^{1}(\Omega)},
$$

which holds true for the weak solution of Dirichlet problems (2.2), we can deduce the following a priori estimate

$$
\|y\|_{H_{0}^{1}(\Omega)} \equiv\left\|(-\Delta)^{-1} u^{*}\right\|_{H_{0}^{1}(\Omega)} \equiv\left\|\nabla(-\Delta)^{-1} u^{*}\right\|_{L^{2}\left(\Omega ; \mathbb{R}^{2}\right)} \leqslant\left\|u^{*}\right\|_{H^{-1}(\Omega)} .
$$

Combining this result with (2.1), we obtain the following chain of inequalities for the dual norm $\|\cdot\|_{H^{-1}(\Omega)}$ in $H^{-1}(\Omega)$ :

$$
\begin{aligned}
\left\|\nabla(-\Delta)^{-1} u^{*}\right\|_{L^{2}\left(\Omega ; \mathbb{R}^{2}\right)} & \leqslant\left\|u^{*}\right\|_{H^{-1}(\Omega)} \\
& \leqslant \sqrt{\int_{\Omega}\left(F_{1}^{2}(x)+F_{2}^{2}(x)\right) d x} \stackrel{\text { by }}{=} \sqrt[(2.3)]{\int_{\Omega}|\nabla y|_{\mathbb{R}^{2}}^{2} d x} \\
& =\|\nabla y\|_{L^{2}\left(\Omega ; \mathbb{R}^{2}\right)}=\left\|\nabla(-\Delta)^{-1} u^{*}\right\|_{L^{2}\left(\Omega ; \mathbb{R}^{2}\right)} .
\end{aligned}
$$

Hence, the standard norm in $H^{-1}(\Omega)$ is equivalent to the following one (see also [27])

$$
\left\|u^{*}\right\|_{H^{-1}(\Omega)}=\left\|\nabla(-\Delta)^{-1} u^{*}\right\|_{L^{2}\left(\Omega ; \mathbb{R}^{2}\right)} .
$$

Remark 2.1. For the our further analysis, we make use of the following relation. Let $y \in H_{0}^{1}(\Omega)$ be a weak solution to Dirichlet problem (2.2). Then

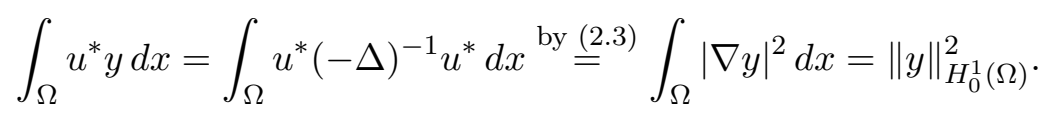

\subsection{Functions of Bounded Variation}

Let $\mathcal{M}\left(\Omega ; \mathbb{R}^{2}\right)$ be the space of all $\mathbb{R}^{2}$-valued Borel measures which is, according to the Riesz theory, the dual of the space $C_{0}\left(\Omega ; \mathbb{R}^{2}\right)$ of all continuous vectorfunctions $\varphi$ with a compact support in $\Omega$, equipped with the uniform norm

$$
\|\varphi\|_{\infty}=\left(\sum_{i=1}^{2} \sup _{x \in \Omega}\left|\varphi_{i}(x)\right|^{2}\right)^{1 / 2} .
$$

Note that $\mathcal{M}\left(\Omega ; \mathbb{R}^{2}\right)$ is isomorphic to the product space $\mathcal{M}^{2}(\Omega):=\prod_{i=1}^{2} \mathcal{M}(\Omega)$ and that $\left(\mu_{1}, \mu_{2}\right) \in \mathcal{M}\left(\Omega ; \mathbb{R}^{2}\right) \Leftrightarrow \mu_{i} \in\left[C_{0}(\Omega)\right]^{*}, i=1,2$. 
By $B V(\Omega)$ we denote the space of all functions $u \in L^{1}(\Omega)$ for which their distributional derivatives are representable by finite Borel measures in $\Omega$, i.e.

$$
\int_{\Omega} u \frac{\partial \phi}{\partial x_{i}} d x=-\int_{\Omega} \phi d D_{i} u, \quad \forall \phi \in C_{0}^{\infty}(\Omega), i=1,2
$$

for some $\mathbb{R}^{2}$-valued measure $D u \in \mathcal{M}^{2}(\Omega)$. It can be shown that $B V(\Omega)$, endowed with the norm

$$
\|u\|_{B V(\Omega)}=\|u\|_{L^{1}(\Omega)}+|D u|(\Omega)
$$

is a Banach space, where

$$
\begin{aligned}
|D u|(\Omega):=\int_{\Omega} d|D u|=\sup \left\{\int_{\Omega} u \operatorname{div} \varphi d x:\right. & \\
& \left.\varphi \in C_{0}^{1}\left(\Omega ; \mathbb{R}^{2}\right),|\varphi(x)| \leqslant 1 \text { for } x \in \Omega\right\}
\end{aligned}
$$

stands for the total variation of $u$ in $\Omega$. It is clear that $|D u|(\Omega)=\int_{\Omega}|\nabla u| d x$ if $u$ is continuously differentiable in $\Omega$.

Remark 2.2. In the similar manner, we can also define the space $B V\left(\Omega ; \mathbb{R}^{M}\right)$ as the space of all vector-valued functions $u: \Omega \rightarrow \mathbb{R}^{M}$ in $L^{1}\left(\Omega ; \mathbb{R}^{M}\right)$ whose distributional derivative $D u$ belongs to the space $\mathcal{M}\left(\Omega ; \mathbb{R}^{M \times 2}\right)$ of $M \times 2$ matrixvalued Borel measures.

According to the Radon-Nikodym theorem, if $u \in B V(\Omega)$ then there exists $\nabla u \in L^{1}\left(\Omega ; \mathbb{R}^{2}\right)$ and a measure $D_{s} u$ singular with respect to the 2-dimensional Lebesgue measure $\mathcal{L}^{2}\left\llcorner\Omega\right.$ restricted to $\Omega$, such that $D u=\nabla u \mathcal{L}^{2}\left\llcorner\Omega+D_{s} u\right.$.

We recall that a sequence $\left\{f_{k}\right\}_{k=1}^{\infty}$ converges weakly* to $f$ in $B V(\Omega)$ if and only if the two following conditions hold (see [4, p.124]): $f_{k} \rightarrow f$ strongly in $L^{1}(\Omega)$ and $D f_{k} \stackrel{*}{\rightarrow} D f$ weakly* in $\mathcal{M}\left(\Omega ; \mathbb{R}^{2}\right)$, i.e.

$$
\lim _{k \rightarrow \infty} \int_{\Omega} \phi d D f_{k}=\int_{\Omega} \phi d D f, \quad \forall \phi \in C_{0}\left(\Omega ; \mathbb{R}^{2}\right),
$$

where, in fact, $D f_{k}=\left(D_{1} f_{k}, D_{2} f_{k}\right) \in \mathcal{M}\left(\Omega ; \mathbb{R}^{2}\right)$ and, therefore, the notation $\int_{\Omega} \phi d D f_{k}$ should be interpreted as follows

$$
\int_{\Omega} \phi d D f_{k}:=\int_{\Omega} \phi_{1} d D_{1} f_{k}+\int_{\Omega} \phi_{2} d D_{2} f_{k} .
$$

Moreover, if $\left\{f_{k}\right\}_{k=1}^{\infty} \subset B V(\Omega)$ converges strongly to some $f$ in $L^{1}(\Omega)$ and $\sup _{k \in \mathbb{N}} \int_{\Omega} d\left|D f_{k}\right|<+\infty$, then (see, for instance, [4] and [5])

$$
\begin{aligned}
& \text { (i) } f \in B V(\Omega) \text { and } \int_{\Omega} d|D f| \leqslant \liminf _{k \rightarrow \infty} \int_{\Omega} d\left|D f_{k}\right| ; \\
& \text { (ii) } f_{k} \stackrel{*}{\rightarrow} f \text { in } B V(\Omega) .
\end{aligned}
$$

So, a simple criterion for weak* convergence can be states as follows (see $[4$, p.125], [18, Theorem 1.19]): 
Proposition 2.1. A sequence $\left\{u_{k}\right\}_{k \in \mathbb{N}} \subset B V(\Omega)$ weakly* converges to $u$ in $B V(\Omega)$ if and only if $\left\{u_{k}\right\}_{k \in \mathbb{N}}$ is bounded in $B V(\Omega)$ and converges to $u$ strongly in $L^{1}(\Omega)$.

The following embedding results for $B V$-function is very useful in connection with variational problem that we study in this paper (see [5, p.378]).

Proposition 2.2. Let $\Omega$ be an open bounded Lipschitz subset of $\mathbb{R}^{2}$. Then the embedding $B V(\Omega) \hookrightarrow L^{2}(\Omega)$ is continuous and the embeddings $B V(\Omega) \hookrightarrow L^{p}(\Omega)$ are compact for all $p$ such that $1 \leqslant p<2$. Moreover, there exists a constant $C_{e m}>0$ which depends only on $\Omega$ and $p$ such that for all $u$ in $B V(\Omega)$,

$$
\left(\int_{\Omega}|u|^{p} d x\right)^{1 / p} \leqslant C_{e m}\|u\|_{B V(\Omega)}, \quad \forall p \in[1,2] .
$$

We also recall the Poincare-Wirtinger inequality: in two dimensional case, there exists a constant $C_{P W}$ such that, for any $u \in B V(\Omega)$, we have

$$
\left\|u-\langle u\rangle_{\Omega}\right\|_{L^{2}(\Omega)} \leqslant C_{P W} \int_{\Omega} d|D u| \leqslant C_{P W}\|u\|_{B V(\Omega)},
$$

where

$$
\langle u\rangle_{\Omega}:=\frac{1}{|\Omega|} \int_{\Omega} u(x) d x
$$

denotes the mean of $u$ in $\Omega$.

By analogy with the theory of Sobolev spaces, the notion of trace operator can be extended for $B V$-functions. Namely, the following result is well-known [5, p. 378]

Theorem 2.2. Let $\Omega$ be an open bounded Lipschitz subset of $\mathbb{R}^{2}$. Let $\partial \Omega$ be its topological boundary. Then there exists a linear continuous map $\gamma_{0}$ from $B V(\Omega)$ onto $L^{1}\left(\partial \Omega, d \mathcal{H}^{1}\right)$ such that $u^{\partial \Omega}:=\gamma_{0}(u)$ for all $u \in B V(\Omega)$, i.e.,

- for all $u$ in $C(\bar{\Omega}) \cap B V(\Omega), \gamma_{0}(u)=\left.u\right|_{\partial \Omega}$;

- the Green's formula holds: $\forall \varphi \in C^{1}\left(\bar{\Omega} ; \mathbb{R}^{2}\right)$,

$$
\int_{\Omega} \varphi d D u=-\int_{\Omega} u \operatorname{div} \varphi d x+\int_{\partial \Omega} \gamma_{0}(u)(\varphi, \nu)_{\mathbb{R}^{2}} d \mathcal{H}^{1}
$$

where $\nu(x)$ is the outer unit normal at $\mathcal{H}^{1}$-almost all $x$ in $\partial \Omega$.

\subsection{Sets of Finite Perimeter}

In this subsection, we remind the main properties of the so-called sets of finite perimeter introduced by $\mathrm{R}$. Caccioppoli in [9]. 
Definition 2.1. Let $E$ be an $\mathcal{L}^{2}$-measurable subset of $\mathbb{R}^{2}$ with finite Lebesgue measure. Let $\chi_{E}$ be its characteristic function. We say that $E$ is a set with finite perimeter in $\Omega$ if $\chi_{E} \in B V(\Omega)$. This means that the distributional gradient $D \chi_{E}$ is a vector-valued measure with finite total variation. The total variation $D \chi_{E} \mid(\Omega)$ is called the perimeter of $E$ in $\Omega$, i.e., $P(E, \Omega)=\left|D \chi_{E}\right|(\Omega)$ and, therefore,

$$
P(E, \Omega)=\sup \left\{\int_{\Omega} \chi_{E} \operatorname{div} \varphi d x: \varphi \in C_{0}^{1}\left(\Omega ; \mathbb{R}^{2}\right),\|\varphi\|_{L^{\infty}\left(\Omega ; \mathbb{R}^{2}\right)} \leqslant 1\right\} .
$$

From the theory of functions of bounded variation it is known that for every set $E$ with finite perimeter in $\Omega$ there exists a vector-valued Radon measure $D \chi_{E}$ such that a generalized Gauss-Green formula holds:

$$
\int_{\Omega} \chi_{E} \operatorname{div} \varphi d x=-\int_{\Omega}\left(\nu_{E}, \varphi\right)_{\mathbb{R}^{2}} d\left|D \chi_{E}\right|
$$

for all $\varphi \in C_{0}^{1}\left(\Omega ; \mathbb{R}^{2}\right)$, where $\nu_{E}$ is the inner unit normal to $E$ and $D \chi_{E}=\nu_{E}\left|D \chi_{E}\right|$ is called the polar decomposition of $D \chi_{E}$.

Since the sets with finite perimeter are not smooth in general, the correct way to represent the measure $D \chi_{E}$ is to introduce the so-called reduced boundary $\partial^{*} E$.

Definition 2.2. Let $E$ be a set of finite perimeter (in $\mathbb{R}^{2}$ ). We say that $x \in \partial^{*} E$ if

1. for every $r>0$ we have $0<$ meas $\left(E \cap B_{r}(x)\right)<$ meas $\left(B_{r}(x)\right)$;

2. there exists the limit

$$
\nu_{E}(x)=\lim _{r \rightarrow 0} \frac{D \chi_{E}\left(B_{r}(x)\right)}{\left|D \chi_{E}\right|\left(B_{r}(x)\right)} \quad \text { and } \quad\left|\nu_{E}(x)\right|=1 .
$$

where the set $\partial^{*} E$ is called the reduced boundary of $E$.

In this way, for every set $E$ of finite perimeter we have

$$
D \chi_{E}=\nu_{E}(x) \mathcal{H}^{1}\left\llcorner\partial^{*} E .\right.
$$

The following properties are well-known:

(a) $P(E, \Omega)=\mathcal{H}^{1}\left(\Omega \cap \partial^{*} E\right) \leqslant \mathcal{H}^{1}(\Omega \cap \partial E)$;

(b) Any sequence of $\mathcal{L}^{2}$-measurable subsets $\left\{E_{k}\right\}_{k \in \mathbb{N}} \subset \Omega$ such that

$$
\sup _{k \in \mathbb{N}} P\left(E_{k}, \Omega\right)<+\infty
$$

admits a subsequence $\left\{E_{k(i)}\right\}_{i \in \mathbb{N}}$ converging in measure in $\Omega$ to some $E \subset \Omega$, i.e., $\left|E_{k(i)} \Delta E\right| \rightarrow 0$ as $i \rightarrow \infty$; 
(c) $E \mapsto P(E, \Omega)$ is lower semicontinuous with respect to the convergence in measure in $\Omega$;

(d) For any two sets $E_{1}$ and $E_{2}$ with finite perimeters in $\Omega$, the relation

$$
P\left(E_{1} \cup E_{2}, \Omega\right) \leqslant P\left(E_{1}, \Omega\right)+P\left(E_{2}, \Omega\right)
$$

holds, with equality holding if only the distance between these sets in Euclidean space $\mathbb{R}^{2}$ is non-zero.

Remark 2.3. Hereinafter, in view of the Lipschitz property of an open bounded domain $\Omega \subset \mathbb{R}^{2}$, it is plausible to suppose that $\Omega$ is the set with finite perimeter and such that $\partial \Omega=\partial^{*} \Omega$.

We recall here some auxiliary results concerning the vector fields $z \in L^{\infty}\left(\Omega ; \mathbb{R}^{2}\right)$ whose divergence in the sense of distribution is an $L^{2}(\Omega)$-function. We denote by $L_{2, d i v}^{\infty}\left(\Omega ; \mathbb{R}^{2}\right)$ the space of all such vector-valued fields $z$. Then the trace of the normal component of the vector field $z \in L_{2, d i v}^{\infty}\left(\Omega ; \mathbb{R}^{2}\right)$ on $\partial \Omega$ can be defined as distribution $\operatorname{Tr}(z, \partial \Omega)$ in the sense of Anzellotti (see [3]). In particular, having assumed that the original domain $\Omega \subset \mathbb{R}^{2}$ is of class $C^{1}$, the trace of the normal component of $z$ on $\partial \Omega$ is the distribution defined as follows

$$
\langle\operatorname{Tr}(z, \partial \Omega), \varphi\rangle:=\int_{\Omega}(z, \nabla \varphi)_{\mathbb{R}^{2}} d x+\int_{\Omega} \varphi \operatorname{div} z d x, \quad \forall \varphi \in C_{0}^{\infty}\left(\mathbb{R}^{2}\right) .
$$

For example, if $z$ is a piecewise continuous vector field that can be extended continuously in $\bar{\Omega}$, then (see [13, p. 22]) $\operatorname{Tr}(z, \partial \Omega)=(z, \nu)_{\mathbb{R}^{2}}$, where $\nu \in \mathbb{R}^{2}$ is the outward unit normal on $\partial \Omega$.

Utilizing the property $\partial \Omega=\partial^{*} \Omega$, we have the following result (the so-called Gauss-Green formula) (see [13, Theorem 5.1] and [21, Proposition 6.12] for the details).

Lemma 2.1. For any $u \in B V(\Omega)$ and $z \in L_{2, d i v}^{\infty}\left(\Omega ; \mathbb{R}^{2}\right)$ there exists a Radone measure on $\Omega$ denoted by $(z, D u)$ and a function $\operatorname{Tr}(z, \partial \Omega) \in L^{\infty}(\partial \Omega)$ such that

$$
\int_{\Omega} u \operatorname{div} z d x+\int_{\Omega} d(z, D u)=\int_{\partial \Omega} \operatorname{Tr}(z, \partial \Omega) u^{\partial \Omega} d \mathcal{H}^{1}
$$

and, for all $\varphi \in C_{0}^{\infty}(\Omega)$,

$$
\langle(z, D u), \varphi\rangle=-\int_{\Omega} u \varphi \operatorname{div} z d x-\int_{\Omega}(u z, \nabla \varphi) d x,
$$

$u^{\partial \Omega} \in L^{1}\left(\partial \Omega, d \mathcal{H}^{1}\right)$ stands for the trace of $u \in B V(\Omega)$ on $\partial \Omega$. The measure $(z, D u)$ and $|(z, D u)|$ are absolutely continuous with respect to $|D u|$ and, for any open $\widetilde{\Omega} \subset \Omega, \forall \varphi \in C_{0}^{\infty}(\widetilde{\Omega})$, and for all Borel sets $\widehat{\Omega} \subset \widetilde{\Omega}$, we have

$$
\begin{gathered}
|\langle(z, D u), \varphi\rangle| \leqslant\|\varphi\|_{L^{\infty}(\widetilde{\Omega})}\|z\|_{L^{\infty}\left(\widetilde{\Omega} ; \mathbb{R}^{2}\right)} \int_{\widetilde{\Omega}} d|D u|, \\
\left|\int_{\widehat{\Omega}} d(z, D u)\right| \leqslant \int_{\widehat{\Omega}} d|(z, D u)| \leqslant\|z\|_{L^{\infty}\left(\widetilde{\Omega} ; \mathbb{R}^{2}\right)} \int_{\widehat{\Omega}} d|D u| .
\end{gathered}
$$


Moreover, it turns out that in this case the following estimate holds true

$$
\|\operatorname{Tr}(z, \partial \Omega)\|_{L^{\infty}(\partial \Omega)} \leqslant\|z\|_{L^{\infty}\left(\Omega ; \mathbb{R}^{2}\right)}
$$

\section{Setting of the Coupled Optimization Problem and Its Previous Analysis}

We begin with the following assumptions:

(i) $\left\{u_{i, d}\right\}_{i=1}^{M} \in L^{2}(\Omega)$ and $\left\{v_{i, d}\right\}_{i=1}^{M} \in L^{2}(\Omega)$ are given collections of distributions;

(ii) $\left\{B_{i}: L^{2}(\Omega) \rightarrow L^{2}(\Omega)\right\}_{i=1}^{M}$ is a collection of linear continuous operators such that

$$
B_{i} \chi_{\Omega} \neq 0 \text { in } L^{2}(\Omega), \quad \forall i=1, \ldots, M ;
$$

(iii) $\left\{V_{i}: B V(\Omega) \rightarrow B V(\Omega)\right\}_{i=1}^{M}$ are, in general, nonlinear sequentially continuous operators in the following sense: if $\left\{u_{k}\right\}_{k \in \mathbb{N}}$ is a sequence in $B V(\Omega)$ such that $u_{k} \stackrel{*}{\rightarrow} u$ in $B V(\Omega)$ as $k \rightarrow \infty$, then $V_{i}\left(u_{k}\right) \rightarrow V_{i}(u)$ strongly in $L^{1}(\Omega)$ for each $i=1, \ldots, M$;

(iv) $\left\{\lambda_{j}>0\right\}_{j=1}^{3}$ are fixed scale parameters.

For the collection of energy functionals

$$
\begin{gathered}
J_{1}(u)=\int_{\Omega} d|D u|+\lambda_{1}\left\|u-u_{1, d}\right\|_{H^{-1}(\Omega)}^{2}+\lambda_{2}\left\|B_{1}(u)-v_{1, d}\right\|_{L^{2}(\Omega)}^{2}, \\
\ldots \ldots \\
J_{M}(u)=\int_{\Omega} d|D u|+\lambda_{1}\left\|u-u_{M, d}\right\|_{H^{-1}(\Omega)}^{2}+\lambda_{2}\left\|B_{M}(u)-v_{M, d}\right\|_{L^{2}(\Omega)}^{2}, \\
J_{0}(E)=\lambda_{3} \int_{\Omega} d\left|D \chi_{E}\right|+\int_{\Omega}\left|V\left(\mathbf{u}^{o p t}\right)-c_{1}\right|^{r} \chi_{E} d x \\
\quad+\int_{\Omega}\left|V\left(\mathbf{u}^{o p t}\right)-c_{2}\right|^{r}\left(1-\chi_{E}\right) d x
\end{gathered}
$$

with $r \geqslant 1$ and

$$
\begin{gathered}
c_{1}=\left\langle V\left(\mathbf{u}^{o p t}\right)\right\rangle_{E}=\frac{1}{|E|} \int_{E} V\left(\mathbf{u}^{o p t}\right) d x, \\
c_{2}=\left\langle V\left(\mathbf{u}^{o p t}\right)\right\rangle_{\Omega \backslash E}=\frac{1}{|\Omega \backslash E|} \int_{\Omega \backslash E} V\left(\mathbf{u}^{o p t}\right) d x,
\end{gathered}
$$

we consider the following coupled variational problem

$$
J_{0}(E) \rightarrow \inf , \quad E \subseteq \Omega .
$$


Here,

$$
\begin{gathered}
\mathbf{u}^{o p t}=\left(u_{1}^{o p t}, \ldots, u_{M}^{o p t}\right), \\
J_{i}\left(u_{i}^{o p t}\right)=\inf _{u \in \Xi} J_{i}(u), \quad i=1, \ldots, M, \\
u_{i}^{o p t} \in \Xi, \quad i=1, \ldots, M, \\
V\left(\mathbf{u}^{o p t}\right)=\sum_{i=1}^{M-1} \alpha_{i} \frac{V_{M}\left(u_{M}^{o p t}\right)}{V_{i}\left(u_{i}^{o p t}\right)+V_{M}\left(u_{M}^{o p t}\right)}, \quad \alpha_{i} \geqslant 0,
\end{gathered}
$$

and the set of feasible solutions $\Xi$ is given by the rule

$$
\Xi=\{u \in B V(\Omega): u(x) \geqslant 0 \text { a.e. in } \Omega\} .
$$

Remark 3.1. If $E^{o p t} \subseteq \Omega$ minimizes the cost functional $J_{0}$, then adding to $E^{\text {opt }}$ any $\mathcal{L}^{2}$-negligible piece, we formally obtain a new optimal solution $E^{\prime} \subseteq \Omega$ because such perturbation of $E^{o p t}$ inside $\Omega$ do not decrease the cost functional $J_{0}$. For this reason, in order to avoid any ambiguity in the sequel, it makes sense to introduce the concept of essential optimal solution [4, p. 319]: it has the property that $\mathcal{L}^{2}\left(E^{\text {opt }} \cap B(x)\right)>0$ for any $x \in E^{\text {opt }}$ and any ball $B(x) \subset \Omega$. As a result, it can be shown that any optimal solution $E^{o p t}$ induces an essential optimal one $E^{\prime}$, with $E^{\prime} \subset E^{o p t}$.

Let us show that the above mentioned variational problem is consistent, that is, there exists a nonempty Borel subset of $E$ of $\Omega$ and a vector-valued function $\mathbf{u}^{o p t}=\left(u_{1}^{o p t}, \ldots, u_{M}^{o p t}\right) \in B V\left(\Omega ; \mathbb{R}^{M}\right)$ such that $J_{0}(E)<+\infty$. With that in mind we begin with the following result (for comparison, we refer to $[1,11,22,24,28]$ ).

Proposition 3.1. For given $\lambda_{1} \geqslant 0, \lambda_{2}>0, u_{i, d} \in L^{2}(\Omega), v_{i, d} \in L^{2}(\Omega)$, and each $i \in\{1, \ldots, M-1\}$, under assumptions (i)-(iv), there exists a unique solution $u_{i}^{\text {opt }}$ of constrained minimization problem (3.9)-(3.10).

Proof. Fixing $i \in\{1, \ldots, M-1\}$, let us show that the corresponding minimization problem (3.9)-(3.10) is consistent, in particular, $J_{i}(u)<+\infty$ for any $u \in \Xi$. Indeed, since $\partial \Omega$ is Lipschitz and we deal with two-dimensional domain $\Omega$, it follows from Poincare inequality (2.9) that $B V(\Omega)$ is continuously embedded into $L^{2}(\Omega)$. In fact, in this particular case we can assert that the injection $B V(\Omega) \hookrightarrow L^{q}(\Omega)$ is compact for all $q \in[1,2)$. On the other hand, by Sobolev embedding theorem we have a continuous injection $H_{0}^{1}(\Omega) \hookrightarrow L^{2}(\Omega)$. Hence, by the duality arguments and Riesz representation theorem, we deduce that $L^{2}(\Omega)=\left[L^{2}(\Omega)\right]^{*} \hookrightarrow\left[H_{0}^{1}(\Omega)\right]^{*}=H^{-1}(\Omega)$ with continuous embedding. Thus, to finalize the remark about consistency, it is enough to observe that $B_{i}(u) \in L^{2}(\Omega)$ for all $u \in B V(\Omega)$. Hence, $J(u)<+\infty$ provided $u \in \Xi$.

Further, we note that the cost functional in (3.9) is non-negative. Hence, the infimum in (3.9)-(3.10) is finite. Let $\left\{u_{i, k}\right\}_{k \in \mathbb{N}}$ be a minimizing sequence for 
(3.9)-(3.10), i.e. $\lim _{k \rightarrow \infty} J_{i}\left(u_{i, k}\right)=\inf _{u \in \Xi} J_{i}(u) \geqslant 0$. Then there exists a constant $C>0$ such that

$$
\begin{gathered}
\sup _{k \in \mathbb{N}}\left(\int_{\Omega}\left|\nabla u_{i, k}\right| d x+\left|D_{s} u_{i, k}\right|(\Omega)\right) \leqslant C, \\
\sup _{k \in \mathbb{N}}\left\|u_{i, k}-u_{i, d}\right\|_{H^{-1}(\Omega)}^{2} \leqslant C, \\
\sup _{k \in \mathbb{N}}\left\|B_{i}\left(u_{i, k}\right)-v_{i, d}\right\|_{L^{2}(\Omega)}^{2} \leqslant C .
\end{gathered}
$$

Now, we prove that there exists a positive constant $M$ such that

$$
\sup _{k \in \mathbb{N}} \int_{\Omega}\left|u_{i, k}\right| d x \leqslant M
$$

With that in mind, we set $w_{i, k}=\frac{1}{|\Omega|} \int_{\Omega} u_{i, k} d x$ and $z_{i, k}=u_{i, k}-w_{i, k}, \forall k \in \mathbb{N}$. Since $u_{i, k}(x) \geqslant 0$ for almost all $x \in \Omega$, it follows that

$$
\int_{\Omega}\left|u_{i, k}\right| d x=|\Omega| w_{i, k}, \quad \int_{\Omega} z_{i, k} d x=0, \text { and } D z_{i, k}=D u_{i, k}, \quad \forall k \in \mathbb{N} .
$$

Hence, (3.12) implies that

$$
\sup _{k \in \mathbb{N}} \int_{\Omega} d\left|D z_{i, k}\right| d x \leqslant C
$$

Then, in view of (2.9), we deduce that

$$
\sup _{k \in \mathbb{N}}\left\|z_{i, k}\right\|_{L^{2}(\Omega)} \leqslant C_{P W} \sup _{k \in \mathbb{N}} \int_{\Omega} d\left|D z_{i, k}\right| \leqslant C_{P W} C
$$

On the other hand, by estimate (3.14) we see that

$$
\begin{aligned}
C & \geqslant \sup _{k \in \mathbb{N}}\left\|B_{i}\left(u_{i, k}\right)-v_{i, d}\right\|_{L^{2}(\Omega)}^{2} \\
& =\sup _{k \in \mathbb{N}}\left\|B_{i}\left(z_{i, k}\right)+B_{i}\left(w_{i, k}\right)-v_{i, d}\right\|_{L^{2}(\Omega)}^{2} \\
& \geqslant \sup _{k \in \mathbb{N}}\left(\left\|B_{i}\left(z_{i, k}\right)-v_{i, d}\right\|_{L^{2}(\Omega)}-\left\|B_{i}\left(w_{i, k}\right)\right\|_{L^{2}(\Omega)}\right)^{2} \\
& \geqslant \sup _{k \in \mathbb{N}}\left[\left(\left\|B_{i}\left(w_{i, k}\right)\right\|_{L^{2}(\Omega)}-2\left\|B_{i}\left(z_{i, k}\right)-v_{i, d}\right\|_{L^{2}(\Omega)}\right)\left\|B_{i}\left(w_{i, k}\right)\right\|_{L^{2}(\Omega)}\right] .
\end{aligned}
$$

Hence,

$$
\begin{aligned}
\sup _{k \in \mathbb{N}}\left\|B_{i}\left(w_{i, k}\right)\right\|_{L^{2}(\Omega)} \leqslant \sup _{k \in \mathbb{N}}\left\|B_{i}\left(z_{i, k}\right)-v_{i, d}\right\|_{L^{2}(\Omega)} & \\
& +\sqrt{\sup _{k \in \mathbb{N}}\left\|B_{i}\left(z_{i, k}\right)-v_{i, d}\right\|_{L^{2}(\Omega)}+C} .
\end{aligned}
$$


Since

$$
\sup _{k \in \mathbb{N}}\left\|B_{i}\left(z_{i, k}\right)-v_{i, d}\right\|_{L^{2}(\Omega)} \leqslant\left\|v_{i, d}\right\|_{L^{2}(\Omega)}+\left\|B_{i}\right\|_{\mathcal{L}\left(B V(\Omega), L^{2}(\Omega)\right.} \sup _{k \in \mathbb{N}}\left\|z_{i, d}\right\|_{B V(\Omega)}
$$

and

$$
\begin{gathered}
\left\|z_{i, d}\right\|_{B V(\Omega)}=\left\|z_{i, d}\right\|_{L^{1}(\Omega)}+\left|D z_{i, k}\right|(\Omega) \leqslant \sqrt{|\Omega|}\left\|z_{i, d}\right\|_{L^{2}(\Omega)}+\left|D z_{i, k}\right|(\Omega) \\
\text { by }(3.17)-(3.18) \\
\leqslant
\end{gathered}
$$

it follows that

$$
\begin{aligned}
\sup _{k \in \mathbb{N}}\left\|B_{i}\left(w_{i, k}\right)\right\|_{L^{2}(\Omega)} \leqslant M^{*}:=(\sqrt{|\Omega|}+1) & C_{P W} C \\
& +\sqrt{(\sqrt{|\Omega|}+1) C_{P W} C+C} .
\end{aligned}
$$

Then, taking into account the definition of $w_{i, k}$ and assumption (ii), we have

$$
\begin{aligned}
\sup _{k \in \mathbb{N}}\left\|B_{i}\left(w_{i, k}\right)\right\|_{L^{2}(\Omega)}^{2} & =\frac{\left\|B_{i}\left(\chi_{\Omega}\right)\right\|_{L^{2}(\Omega)}^{2}}{|\Omega|} \sup _{k \in \mathbb{N}}\left[\int_{\Omega} u_{i, k} d x\right] \\
& \text { by } \stackrel{(3.19)}{\leqslant}\left(M^{*}\right)^{2} .
\end{aligned}
$$

Hence, due to condition (3.1), we obtain

$$
\sup _{k \in \mathbb{N}}\left\|u_{i, k}\right\|_{L^{1}(\Omega)}=\sup _{k \in \mathbb{N}} \int_{\Omega}\left|u_{i, k}\right| d x \leqslant \frac{|\Omega|}{\left\|B_{i}\left(\chi_{\Omega}\right)\right\|_{L^{2}(\Omega)}^{2}}\left(M^{*}\right)^{2} .
$$

Combining this fact with estimates (3.12)-(3.13), we finally deduce that the minimizing sequence $\left\{u_{i, k}\right\}_{k \in \mathbb{N}}$ is bounded in $B V(\Omega)$ and $H^{-1}(\Omega)$. Therefore (see [18, Theorem 1.19]), there exists a subsequence, still denoted by the same index, and elements $u_{i}^{0} \in B V(\Omega)$ and $u_{i}^{*} \in H^{-1}(\Omega)$ such that $u_{i, k} \rightarrow u_{i}^{0}$ strongly in $L^{1}(\Omega)$, $u_{i, k}(x) \rightarrow u_{i}^{0}(x)$ almost everywhere in $\Omega, D u_{i, k} \stackrel{*}{\rightarrow} D u_{i}^{0}$ weakly* in $\mathcal{M}\left(\Omega ; \mathbb{R}^{2}\right)$, and $u_{i, k} \rightarrow u_{i}^{*}$ weakly in $H^{-1}(\Omega)$ as $k \rightarrow \infty$.

Let us show that, in fact, $u_{i}^{0}=u_{i}^{*}$ almost everywhere in $\Omega$. Indeed, as follows from continuity of the embedding $B V(\Omega) \hookrightarrow L^{2}(\Omega)$, the sequence $\left\{u_{i, k}\right\}_{k \in \mathbb{N}}$ is bounded in $L^{2}(\Omega)$. Then, pointwise convergence $u_{i, k} \rightarrow u_{i}^{0}$ in $\Omega$ implies the weak convergence $u_{i, k} \rightarrow u_{i}^{0}$ in $L^{2}(\Omega)$ by Egoroff's theorem. Since $L^{2}(\Omega) \hookrightarrow H^{-1}(\Omega)$ is the compact embedding, it follows that $u_{i, k} \rightarrow u_{i}^{0}$ strongly in $H^{-1}(\Omega)$. Hence, $u_{i}^{0}=u_{i}^{*}$ in $\Omega$ by uniqueness of the weak limit in reflexive Banach space.

Utilizing these properties together with inequality (2.8) and lower semicontinuity of $L^{2}$-norm with respect to the weak convergence, we get

$$
\begin{aligned}
\liminf _{k \rightarrow \infty}\left\|B_{i}\left(u_{i, k}\right)-v_{i, d}\right\|_{L^{2}(\Omega)}^{2} & \geqslant\left\|B_{i}\left(u_{i}^{0}\right)-v_{i, d}\right\|_{L^{2}(\Omega)}^{2}, \\
\liminf _{k \rightarrow \infty} \int_{\Omega} d\left|D u_{i, k}\right| & \geqslant \int_{\Omega} d\left|D u_{i}^{0}\right|, \\
\lim _{k \rightarrow \infty}\left\|u_{i, k}-u_{2, d}\right\|_{H^{-1}(\Omega)}^{2} & =\left\|u_{i}^{0}-u_{2, d}\right\|_{H^{-1}(\Omega)}^{2} .
\end{aligned}
$$


As a result, it follows from the above consideration that

$$
J_{i}\left(u_{i}^{0}\right) \leqslant \liminf _{k \rightarrow \infty} J_{i}\left(u_{i, k}\right)=\lim _{k \rightarrow \infty} J_{i}\left(u_{i, k}\right)=\inf _{u \in \Xi} J_{i}(u) .
$$

Since the set of feasible solutions $\Xi$ is convex and closed in $B V(\Omega)$, by Mazur's theorem we have that this set is sequentially closed with respect to the weak* convergence in $B V(\Omega)$. Hence, $u_{i}^{0} \geqslant 0$ almost everywhere in $\Omega$. Thus, $u_{i}^{0} \in \Xi$ and $u_{i}^{0}$ is a minimizer for constrained minimization problem (3.9)-(3.10).

It remains to show that $u_{i}^{0}$ is a unique minimizer for this problem. Indeed, let us assume the converse. Let $u_{i}^{*} \in \Xi$ and $v_{i}^{*} \in \Xi$ be two minimizers for the problem (3.9)-(3.10). By the strict convexity of norms $\|\cdot\|_{L^{2}(\Omega)}$ and $\|\cdot\|_{H^{-1}(\Omega)}$ (see $(2.6)$ ), we obviously have

$$
J_{i}\left(\frac{1}{2} u_{i}^{*}+\frac{1}{2} v_{i}^{*}\right)<\frac{1}{2} J_{i}\left(u_{i}^{*}\right)+\frac{1}{2} J_{i}\left(v_{i}^{*}\right)=\inf _{u \in \Xi} J_{i}(u)
$$

which brings us into conflict with the initial assumptions. Hence, $J_{i}\left(u_{i}^{*}\right)=J_{i}\left(v_{i}^{*}\right)$ and, as a consequence, we obtain

$$
B_{i}\left(u_{i}^{*}\right)=B_{i}\left(v_{i}^{*}\right) \text { in } L^{2}(\Omega), u_{i}^{*}=v_{i}^{*} \text { in } H^{-1}(\Omega) .
$$

Even if the operator $B_{i} \in \mathcal{L}\left(B V(\Omega), L^{2}(\Omega)\right.$ is not injective, equalities (3.21) imply that $u_{i}^{*}=v_{i}^{*}$ almost everywhere in $\Omega$. Thus, $u_{i}^{0}$ is a unique minimizer to the problem (3.9)-(3.10). The proof is complete.

Our next step is to study variational problem (3.7). To begin with, let us show that this problem is consistent. It would be rather provocative to assert that any measurable subset $E$ of $\Omega$ can be considered as a feasible solution to the problem (3.7). Therefore, it is reasonable to define the corresponding set of feasible solutions $\Xi_{0}$ as follows: $E \in \Xi_{0}$ if and only if, for a given distribution $\mathbf{u}^{\text {opt }} \in B V\left(\Omega ; \mathbb{R}^{M}\right)$, we have

$$
E \subset \Omega, E \text { is a measurable set, and } J_{0}(E)<+\infty .
$$

Remark 3.2. To show that variational problem (3.7) is consistent, i.e. $\Xi_{0} \neq \emptyset$, it is enough to make use of properties (a)-(d) and the fact that the functions $u_{i}^{\text {opt }} \in B V(\Omega), i=1, \ldots, M$, are finite $\mathcal{L}^{2}$-almost everywhere in $\Omega$. Indeed, having set $\Omega_{i, n}=\left\{x \in \Omega:\left|u_{i}^{o p t}(x)\right| \geqslant n\right\}$, we see that $n \chi_{\Omega_{i, n}}(x) \leqslant n \leqslant\left|u_{i}^{o p t}(x)\right|$, $\forall x \in \Omega_{i, n}$. Hence, using the monotonicity of Lebesgue integral, we have

$$
n \mathcal{L}^{2}\left(\Omega_{i, n}\right)=\int_{\Omega} n \chi_{\Omega_{i, n}} d x \leqslant \int_{\Omega_{i, n}}\left|u_{i}^{o p t}\right| d x \leqslant \int_{\Omega}\left|u_{i}^{o p t}\right| d x \leqslant\left\|u_{i}^{o p t}\right\|_{B V(\Omega)},
$$

so, $\mathcal{L}^{2}\left(\Omega_{i, n}\right) \leqslant\left\|u_{i}^{o p t}\right\|_{B V(\Omega)} / n$. Let $\Omega_{i, \infty}:=\bigcap_{n \in \mathbb{N}} \Omega_{i, n}$. Then $x \in \Omega_{i, \infty}$ if and only if $\left|u_{i}^{o p t}(x)\right|=\infty$. Since

$$
0 \leqslant \mathcal{L}^{2}\left(\Omega_{i, \infty}\right) \leqslant \mathcal{L}^{2}\left(\Omega_{i, n}\right) \leqslant \frac{\left\|u_{i}^{o p t}\right\|_{B V(\Omega)}}{n},
$$


and letting $n \rightarrow \infty$, it follows that $\mathcal{L}^{2}\left(\Omega_{i, \infty}\right)=0$, that is, each of functions $u_{i}^{o p t} \in B V(\Omega)$ is finite $\mathcal{L}^{2}$-almost everywhere in $\Omega$. As a result, we have

$$
\frac{V_{M}\left(u_{M}^{o p t}\right)}{V_{i}\left(u_{i}^{o p t}\right)+V_{M}\left(u_{M}^{o p t}\right)} \leqslant 1 \quad \mathcal{L}^{2} \text {-a.e.in } \Omega, \forall i=\overline{1, M-1} .
$$

Then, we immediately deduce from (3.11) that $V\left(\mathbf{u}^{\text {opt }}\right) \in L^{\infty}(\Omega)$, and therefore, the last two terms in the energy functional $J_{0}$ (see (3.4)) are well defined for any $\mathcal{L}^{2}$-measurable subset $E \subset \Omega$. It remains to notice that the inclusion $\chi_{E} \in B V(\Omega)$ is equivalent to the condition $\left|D \chi_{E}\right|(\Omega)<+\infty$. Since $P(E, \Omega)=\left|D \chi_{E}\right|(\Omega)$, it follows that $J_{0}(E)<+\infty$ if and only if $E$ is a subset of $\Omega$ and it has a finite perimeter in $\Omega$. In view of these observations, the set of feasible solutions $\Xi_{0}$ to the problem (3.7) can be redefined as follows

$$
\Xi_{0}=\{E \subseteq \Omega: P(E, \Omega)<+\infty\} .
$$

Remark 3.3. It should be notice that the class $\Xi_{0}$ of admissible domains does not have any linear or convex structure, so in optimization problem (3.7) it is meaningless to speak of convex functionals and similar notions. So, non-uniqueness of its solutions is the standard situation in this case.

To clarify this option, we give the following observation.

Proposition 3.2. For given $\mathbf{u}^{\text {opt }}=\left(u_{1}^{o p t}, \ldots, u_{M}^{\text {opt }}\right)$, let $E^{o p t} \in \Xi_{0}$ be an essential optimal solution of minimization problem (3.7). Then the set $E^{\text {opt }}$ is unique in the following sense: there does not exist a subset $E^{0} \in \Xi_{0}$ such that $E^{0} \in \Xi_{0}$ is an essential optimal solution,

$$
J_{0}\left(E^{0}\right)=\inf _{E \in \Xi_{0}} J_{0}(E), \quad \mathcal{L}^{2}\left(E^{o p t} \cap E^{0}\right)>0 .
$$

Proof. Let us assume the converse. Let $E^{0} \in \Xi_{0}$ be a subset of $\Omega$ such that $E^{0}$ is an essential optimal solution of (3.7) and there exists a subset $E^{*} \subseteq X^{o p t} \cap E^{0}$ such that $\mathcal{L}^{2}\left(E^{*}\right)>0$. Let $\chi_{E^{*}}$ be the corresponding characteristic function. Then it is clear that

$$
\begin{aligned}
& \chi_{E^{*}}<\frac{1}{2} \chi_{E^{o p t}}+\frac{1}{2} \chi_{E^{0}} \text { in } \Omega \backslash E^{*}, \\
& \chi_{E^{*}}=\frac{1}{2} \chi_{E^{o p t}}+\frac{1}{2} \chi_{E^{0}} \text { in } E^{*} .
\end{aligned}
$$

Then, taking into account representation (3.4) and the well-know inequality

$$
\sup _{\theta \in \Theta}(f(\theta)+g(\theta)) \leqslant \sup _{\theta \in \Theta} f(\theta)+\sup _{\theta \in \Theta} g(\theta),
$$

we deduce from (2.7) the following relation

$$
J_{0}\left(E^{*}\right)<\frac{1}{2} J_{0}\left(E^{o p t}\right)+\frac{1}{2} J_{0}\left(E^{0}\right)=\inf _{E \in \Xi_{0}} J_{0}(E),
$$

which leads us into conflict with optimality of $E^{o p t}$ and $E^{0}$. 
Theorem 3.1. Let $\mathbf{u}^{o p t}=\left(u_{1}^{o p t}, \ldots, u_{M}^{o p t}\right)$ be a vector-valued minimizer to the constrained minimization problems (3.9)-(3.10). Then there exists a subset $E^{\text {opt }} \subset$ $\Omega$, with a finite perimeter in $\Omega$, such that

$$
J_{0}\left(E^{o p t}\right)=\inf _{E \in \Xi_{0}} J_{0}(E)
$$

Proof. Since $0 \leqslant J_{0}(E)<+\infty$ for all $E \in \Xi_{0}$, it follows that there exists a non-negative value $\mu \geqslant 0$ such that $\mu=\inf _{E \in \Xi_{0}} J_{0}(E)$. Let $\left\{E_{k}\right\}_{k \in \mathbb{N}} \subset \Omega$ be a minimizing sequence to the problem (3.7), i.e.

$$
E_{k} \in \Xi_{0}, \quad \forall k \in \mathbb{N}, \quad \text { and } \quad \lim _{k \rightarrow \infty} J_{0}\left(E_{k}\right)=\mu .
$$

So, we can suppose that $J_{0}\left(E_{k}\right) \leqslant \mu+1$ for all $k \in \mathbb{N}$. Then

$$
\sup _{k \in \mathbb{N}}\left|D \chi_{E_{k}}\right|(\Omega) \leqslant \frac{1}{\lambda_{3}} \sup _{k \in \mathbb{N}} J_{0}\left(E_{k}\right) \leqslant \frac{\mu+1}{\lambda_{3}}<+\infty .
$$

Therefore, $\left\{\chi_{E_{k}}\right\}_{k \in \mathbb{N}}$ is a bounded sequence in $B V(\Omega)$ and, without loss of generality, we can suppose that there exists a subsequence of $\left\{\chi_{E_{k}}\right\}_{k \in \mathbb{N}}$ (still denoted by the same index) and a function $\chi \in B V(\Omega) \cap L^{\infty}(\Omega)$ such that

$$
\begin{aligned}
& \text { (j) } \chi_{E_{k}} \stackrel{*}{\rightarrow} \chi \text { in } L^{\infty}(\Omega), \chi_{E_{k}} \rightarrow \chi \text { in } L^{1}(\Omega), \\
& (j j) \int_{\Omega} d|D \chi| \leqslant \liminf _{k \rightarrow \infty} \int_{\Omega} d\left|D \chi_{E_{k}}\right| ; \\
& (j j j) \chi_{E_{k}}(x) \rightarrow \chi(x) \text { a.e.in } \Omega, \\
& (j v) D \chi_{E_{k}} \stackrel{*}{\rightarrow} D \chi \text { in } \mathcal{M}\left(\Omega ; \mathbb{R}^{2}\right) .
\end{aligned}
$$

Let us show that, in fact, there exists a subset $E^{\text {opt }} \in \Xi_{0}$ such that $\chi$ is the characteristic function of $E^{o p t}$, i.e. $\chi=\chi_{E^{o p t}}$. Indeed, the element $\chi \in B V(\Omega)$ is the characteristic function of a domain if the identity $\chi(x)(1-\chi(x))=0$ holds true almost everywhere in $\Omega$. Since the equalities

$$
\chi_{E_{k}}(x)\left(1-\chi_{E_{k}}(x)\right)=0 \quad \text { a.e. in } \Omega
$$

are satisfied for all $k \in \mathbb{N}$, then passing to the limit in both sides of (3.26) as $k \rightarrow \infty$, we deduce from (j)-property that the same identity holds for the limit function $\chi \in L^{\infty}(\Omega)$. Hence, we can suppose that $\chi=\chi_{E^{\text {opt }}}$ for some measurable subset $E^{\text {opt }} \subseteq \Omega$. In order to establish the inclusion $E^{o p t} \in \Xi_{0}$, it remains to utilize estimate (3.24) together with the lower semi-continuity property (jj). As a result, we obtain:

$$
P\left(E^{o p t}, \Omega\right)=|D \chi|(\Omega) \leqslant \liminf _{k \rightarrow \infty}\left|D \chi_{E_{k}}\right|(\Omega) \leqslant \frac{\mu+1}{\lambda_{3}}
$$

and, therefore, $E^{o p t} \in \Xi_{0}$. 
Thus, in view of compactness properties of minimizing sequence $\left\{\chi_{E_{k}}\right\}_{k \in \mathbb{N}}$ with respect to the weak* convergence in $B V(\Omega)$, we arrive at the following obvious relation

$$
\begin{aligned}
\inf _{E \in \Xi_{0}} J_{0}(E)= & \lim _{k \rightarrow \infty} J_{0}\left(E_{k}\right) \stackrel{\text { by }}{\geqslant} \int_{\Omega}^{3.25)}\left|V\left(\mathbf{u}^{o p t}\right)-c_{1}\right|^{r} \chi_{E^{o p t}} d x \\
& +\int_{\Omega}\left|V\left(\mathbf{u}^{o p t}\right)-c_{2}\right|^{r}\left(1-\chi_{E^{o p t}}\right) d x+\lambda_{3} \int_{\Omega} d\left|D \chi_{E^{o p t}}\right| \\
= & J_{0}\left(E^{o p t}\right) \geqslant \inf _{E \in \Xi_{0}} J_{0}(E),
\end{aligned}
$$

Thus, $E^{o p t} \subseteq \Omega$ is a solution of variational problem (3.7).

Remark 3.4. As was mentioned in Remark 3.1, we can always suppose that $E^{\text {opt }} \subseteq$ $\Omega$ is an essential optimal solution to variational problem (3.7). Moreover, since any minimizing sequence $\left\{E_{k}\right\}_{k \in \mathbb{N}} \subset \Omega$ is such that $\left\{\chi_{E_{k}}\right\}_{k \in \mathbb{N}}$ is uniformly bounded in $L^{\infty}(\Omega)$, its weak* cluster point $\chi$ in $B V(\Omega)$ is, in fact, an element of the class of special $B V$-functions $S B V(\Omega)$ (see [4, Theorem 4.8]), i.e., the Cantor part $D^{c} \chi$ of its derivative $D \chi$ is zero. In particular, we have the following implication: if $\chi=\chi_{E}, \mathcal{L}^{2}(E)<+\infty$, and $0<P(E, \Omega)<\infty$, then $\chi \in S B V(\Omega)$ but $\chi$ is not, in general, a Sobolev function, i.e., $\chi \notin W^{1,1}(\Omega)$.

Remark 3.5. In view of the well-known fact that the map $\mathbb{R}^{2} \supset E \rightarrow \mathcal{H}^{1}(\partial E)$ is not lower semicontinuous with respect to any compact topology (see [6, Section 4.2] for counter-example and other details), the original variational problem (3.7) can not be replaced by the following one: $J_{0}^{*}(E) \rightarrow$ inf for $E \subset \Omega$, where

$$
J_{0}^{*}(E)=\mathcal{H}^{1}(\partial E)+\int_{\Omega}\left|V\left(\mathbf{u}^{o p t}\right)-c_{1}\right|^{r} \chi_{E} d x+\int_{\Omega}\left|V\left(\mathbf{u}^{o p t}\right)-c_{2}\right|^{r}\left(1-\chi_{E}\right) d x .
$$

In fact, a remarkable result of Federer (see [17, Theorem 4.5.11]) shows that any Borel subset $E \subset \Omega$ satisfying $\mathcal{H}^{1}\left(\Omega \cap \partial^{*} E\right)<+\infty$ has a finite perimeter $P(E, \Omega)=\left|D \chi_{E}\right|(\Omega)$ in $\Omega$. However, equality $P(E, \Omega)=\mathcal{H}^{1}\left(\Omega \cap \partial^{*} E\right)$ holds if only $E$ has a Lipschitz boundary.

\section{Optimality Conditions}

In this section, following in general aspects the technique of Temam for the problem of minimal surfaces [14] and duality results from [15], we derive necessary optimality conditions in order to characterize the solution

$$
\left.\left(\mathbf{u}^{o p t}, E^{o p t}\right)=\left(u_{1}^{o p t}, \ldots, u_{M}^{o p t}, E^{o p t}\right)\right) \in \Xi^{M} \times \Xi_{0}
$$

of the coupled variational problem (3.7)-(3.11).

We begin with the intermediate optimization problem (3.9)-(3.10). 


\subsection{Optimality Conditions for Constrained Minimization Problem (3.9)-(3.10)}

We assume that, for given $i \in\{1, \ldots, M-1\}, \lambda_{1} \geqslant 0, \lambda_{2}>0, u_{i, d} \in L^{2}(\Omega)$, and $v_{i, d} \in L^{2}(\Omega)$, condition (ii) holds true. For our further analysis, we reformulate problem (3.7)-(3.11) as an equivalent problem on the space $X:=L^{2}(\Omega)$. For this reason we define the following functionals on $X$ :

$$
\begin{aligned}
& E_{1}(u)= \begin{cases}\int_{\Omega} d|D u|, & u \in B V(\Omega), \\
+\infty, & u \in L^{2}(\Omega) \backslash B V(\Omega) .\end{cases} \\
& E_{2}(u)=\lambda_{1}\left\|u-u_{i, d}\right\|_{H^{-1}(\Omega)}^{2}, \\
& E_{3}(u)=\lambda_{2}\left\|B_{i}(u)-v_{i, d}\right\|_{L^{2}(\Omega)}^{2}, \\
& G(u)=\int_{\Omega}(u-|u|)^{2} d x .
\end{aligned}
$$

Notice that all indicated functional are well-defined on $X$. In view of this, we extend the energy functional $J_{i}$ to the entire set $L^{2}(\Omega)$ by the rule

$$
\widehat{J}_{i}(u)=E_{1}(u)+E_{2}(u)+E_{3}(u) \quad \forall u \in L^{2}(\Omega) .
$$

Then it is clear that a minimizer $u_{i}^{o p t} \in \Xi$ of (3.7)-(3.11) is also a minimizer of the modified problem

$$
\widehat{J}_{i}(u) \longrightarrow \inf , \quad u \in L^{2}(\Omega), \quad G(u)=0,
$$

because the inclusion $u \in \Xi$ is equivalent to the consistency condition of the problem (4.6), i.e.

$$
u \in \Xi \quad \Leftrightarrow \quad \widehat{J_{i}}(u)<+\infty \text { and } G(u)=0 .
$$

A fundamental difficulty that typically appears in deriving of necessary conditions for minimizers of a constrained minimization problem (4.6), is the lack in differentiability of the energy functional (4.5) and the functional $G(u)$ in the side condition. Since the functionals $\widehat{J}_{i}: B V(\Omega) \rightarrow \mathbb{R}$ and $G: L^{2}(\Omega) \rightarrow \mathbb{R}$ are convex, a necessary condition for a minimizer $u_{i}^{\text {opt }} \in \Xi$ should employ the convex subdifferentials $\partial \widehat{J}_{i}\left(u_{i}^{o p t}\right)$ and $\partial G\left(u_{i}^{o p t}\right)$ which are some subsets of the dual spaces $[B V(\Omega)]^{*}$ and $\left[L^{2}(\Omega)\right]^{*}=L^{2}(\Omega)$, respectively. In spite of the fact that almost nothing is known about the structure of $[B V(\Omega)]^{*}$ (see [4] for the details), we have the continuous embedding $B V(\Omega) \hookrightarrow L^{2}(\Omega)$. Therefore, the elements of $\partial \widehat{J}_{i}\left(u_{i}^{o p t}\right)$ can be evaluated in $L^{2}(\Omega)$ because, by the duality arguments, we have $\left[L^{2}(\Omega)\right]^{*} \hookrightarrow[B V(\Omega)]^{*}$.

We begin with the following technical results.

Proposition 4.1. Let $u_{i, d} \in L^{2}(\Omega)$ be a given distribution. Then the functional $E_{2}: L^{2}(\Omega) \rightarrow \mathbb{R}$ is convex and continuously differentiable on $L^{2}(\Omega)$ with

$$
\begin{aligned}
E_{2}^{\prime}(u)[h]=2 \lambda_{1}\left((-\Delta)^{-1}\left(u-u_{i, d}\right), h\right)_{L^{2}(\Omega)} & \\
= & \left\langle E_{2}^{\prime}(u), h\right\rangle_{X^{*} ; X}, \forall h \in L^{2}(\Omega) .
\end{aligned}
$$


Proof. Let $u \in L^{2}(\Omega)$ be a fixed element. Then, for any direction $h \in L^{2}(\Omega)$, we have $z:=(-\Delta)^{-1} h \in H_{0}^{1}(\Omega)$ and, therefore,

$$
\begin{aligned}
& \lambda_{1}^{-1}\left(E_{2}(u+h)-E_{2}(u)\right) \stackrel{\text { by }}{\stackrel{(2.6)}{=}\left\|\nabla(-\Delta)^{-1}\left(u+h-u_{i, d}\right)\right\|_{L^{2}\left(\Omega ; \mathbb{R}^{2}\right)}^{2}} \\
&-\left\|\nabla(-\Delta)^{-1}\left(u-u_{i, d}\right)\right\|_{L^{2}\left(\Omega ; \mathbb{R}^{2}\right)} \\
&= 2\left(\nabla(-\Delta)^{-1}\left(u-u_{i, d}\right), \nabla(-\Delta)^{-1} h\right)_{L^{2}\left(\Omega ; \mathbb{R}^{2}\right)} \\
&+\left\|\nabla(-\Delta)^{-1} h\right\|_{L^{2}\left(\Omega ; \mathbb{R}^{2}\right)}^{2} .
\end{aligned}
$$

Hence, by (2.6) and the Green formula, we get

$$
\begin{aligned}
\lambda_{1}^{-1}\left(E_{2}(u+h)-\right. & \left.E_{2}(u)\right) \\
& =-2 \int_{\Omega} \operatorname{div}\left[\nabla(-\Delta)^{-1}\left(u-u_{i, d}\right)\right](-\Delta)^{-1} h d x+\|h\|_{H^{-1}(\Omega)}^{2} \\
& =2 \int_{\Omega}(-\Delta)(-\Delta)^{-1}\left(u-u_{i, d}\right)(-\Delta)^{-1} h d x+\|h\|_{H^{-1}(\Omega)}^{2} \\
& =2\left(u-u_{i, d},(-\Delta)^{-1} h\right)_{L^{2}(\Omega)}+\|h\|_{H^{-1}(\Omega)}^{2} \\
& =2\left((-\Delta)^{-1}\left(u-u_{i, d}\right), h\right)_{L^{2}(\Omega)}+o\left(\|h\|_{L^{2}(\Omega)}\right) .
\end{aligned}
$$

Since $2(-\Delta)^{-1}\left(u-u_{i, d}\right) \in \mathcal{L}(X, X)$, it follows that $E_{2}: L^{2}(\Omega) \rightarrow \mathbb{R}$ is Fréchet differentiable at a given $u \in L^{2}(\Omega)$ with the representation (4.7) for its derivative.

Proposition 4.2. Let $v_{i, d} \in L^{2}(\Omega)$ be a given distribution. Then the functional $E_{3}: L^{2}(\Omega) \rightarrow \mathbb{R}$ is convex and continuously differentiable on $L^{2}(\Omega)$ with

$$
\begin{aligned}
E_{3}^{\prime}(u)[h]=2 \lambda_{2}\left(B_{i}^{*}\left(B_{i}(u)-v_{i, d}\right), h\right)_{L^{2}(\Omega)} & \\
& =\left\langle E_{3}^{\prime}(u), h\right\rangle_{X^{*} ; X}, \quad \forall h \in L^{2}(\Omega),
\end{aligned}
$$

where $B_{i}^{*} \in \mathcal{L}(X, X)$ stands for the adjoint operator.

Proof. Fixing arbitrary elements $u \in L^{2}(\Omega)$ and $h \in L^{2}(\Omega)$, we get

$$
\begin{aligned}
\lambda_{2}^{-1} & \left(E_{3}(u+h)-E_{3}(u)\right) \\
& =\left\|B_{i}(u+h)-v_{i, d}\right\|_{L^{2}(\Omega)}^{2}-\left\|B_{i}(u)-v_{i, d}\right\|_{L^{2}(\Omega)}^{2} \\
& =2\left(B_{i}(u)-v_{i, d}, B_{i}(h)\right)_{L^{2}(\Omega)}+\left\|B_{i}(h)\right\|_{L^{2}(\Omega)}^{2} \\
& =2\left(B_{i}^{*}\left(B_{i}(u)-v_{i, d}\right), h\right)_{L^{2}(\Omega)}+o\left(\|h\|_{L^{2}(\Omega)}\right),
\end{aligned}
$$

which obviously leads us to the desired conclusion.

Before proceeding further, we recall the definition of the subdifferential $\partial F(u)$ of a convex proper functional $F: X \rightarrow \mathbb{R} \cup+\infty$ at some element $u \in X$. Setting 
$X=L^{2}(\Omega)$, we see that $X^{*}=X$. Then, for given $u \in X$, an element $\xi \in X$ belongs to $\partial F(u)$ if and only if, $\forall v \in X$,

$$
F(u) \in \mathbb{R} \text { and } F(u)-\int_{\Omega} \xi u d x \leqslant F(v)-\int_{\Omega} \xi v d x .
$$

Thus, $\xi \in \partial F(u)$ if $u$ is a minimizer on $X$ of the following variational problem

$$
\inf _{u \in X}\left[F(v)-\int_{\Omega} \xi v d x\right]
$$

Proposition 4.3. The functional $G: L^{2}(\Omega) \rightarrow \mathbb{R}$ is convex, lower semicontinuous with respect to the weak convergence in $L^{2}(\Omega)$, and Gâteaux differentiable on $L^{2}(\Omega)$ with

$$
G^{\prime}(u)[h]=4 \int_{\Omega}(u-|u|) h d x, \quad \forall h \in L^{2}(\Omega) .
$$

Proof. Since convexity and the lower semicontinuity property of $G: L^{2}(\Omega) \rightarrow \mathbb{R}$ are the direct consequence of (4.4), it follows that this functional has a nonempty subdifferential at each point $u \in L^{2}(\Omega)$. Let us assume that $u$ is a nonzero element of $L^{2}(\Omega)$. Let $\xi \in \partial G(u)$. Then, by definition of subgradient, we have

$$
G(v) \geqslant G(u)+\int_{\Omega} \xi(v-u) d x, \quad \forall v \in L^{2}(\Omega) .
$$

Setting $v=u+\lambda z$ for any $z \in L^{2}(\Omega)$ and $\lambda>0$, we obtain

$$
\begin{aligned}
\int_{\Omega} \xi z d x \leqslant & \frac{G(u+\lambda z)-G(u)}{\lambda} \\
= & \frac{1}{\lambda}\left[2 \int_{\Omega}(u+\lambda z)^{2} d x-2 \int_{\Omega}(u+\lambda z)|u+\lambda z| d x-\int_{\Omega}(u-|u|)^{2} d x\right] \\
= & \frac{1}{\lambda}\left[4 \lambda \int_{\Omega} u z d x+2 \lambda^{2} \int_{\Omega} z^{2} d x-2 \lambda \int_{\Omega} z|u+\lambda z| d x\right. \\
& \left.-2 \int_{\Omega} u \frac{\lambda^{2} z^{2}-2 \lambda z u}{|u|+|u+\lambda z|} d x\right] \\
& \stackrel{\lambda \rightarrow 0}{\rightarrow} 4 \int_{\Omega}(u-|u|) z d x .
\end{aligned}
$$

Hence,

$$
\int_{\Omega}[4(u-|u|)-\xi] z d x \geqslant 0, \quad \forall z \in L^{2}(\Omega)
$$

and by choosing $z=-[4(u-|u|)-\xi]$, we deduce that

$$
\partial G(u)=\{4(u-|u|)\}, \quad \forall u \not \equiv 0 \text { in } L^{2}(\Omega) .
$$

It remains to consider the case $u \equiv 0$. Letting $\xi \in \partial G(0)$, we obtain

$$
G(v) \geqslant \int_{\Omega} \xi v d x, \quad \forall v \in L^{2}(\Omega)
$$


or in other terms

$$
\int_{\Omega}\left[2 v^{2}-2 v|v|-\xi v\right] d x \geqslant 0, \quad \forall v \in L^{2}(\Omega) .
$$

Since the fulfillment of this relation for all $v \in L^{2}(\Omega)$ is possible if only $\xi=0$ in $L^{2}(\Omega)$, it follows from (4.11) that $\partial G(u)$ is a singleton for all $u \in L^{2}(\Omega)$. Hence,

$$
\partial G(u)=\left\{G^{\prime}(u)\right\}=\{4(u-|u|)\}, \quad \forall u \in L^{2}(\Omega) .
$$

Our next intension is to define the structure of subdifferential for the functional $E_{1}: L^{2}(\Omega) \rightarrow \mathbb{R} \cup+\infty$ given by the rule (4.1).

Proposition 4.4. The functional $E_{1}: L^{2}(\Omega) \rightarrow \mathbb{R} \cup+\infty$ is convex, lower semicontinuous, and positively homogeneous of degree 1 on $L^{2}(\Omega)$. Moreover, for a given function $u \in L^{2}(\Omega), \xi \in \partial E_{1}(u)$ if and only if there is a vector-valued field $z \in L_{2, \text { div }}^{\infty}\left(\Omega ; \mathbb{R}^{2}\right)$ with zero trace of the normal component $\operatorname{Tr}(z, \partial \Omega)$ such that

$$
\begin{gathered}
\|z\|_{L^{\infty}\left(\Omega ; \mathbb{R}^{2}\right)} \leqslant 1, \quad \xi=-\operatorname{div} z \in L^{2}(\Omega), \\
E_{1}(u)=(\xi, u)_{L^{2}(\Omega)}=-\int_{\Omega} u \operatorname{div} z d x .
\end{gathered}
$$

In addition, if $E_{1}(u)<+\infty$, then $\|z\|_{L^{\infty}\left(\Omega ; \mathbb{R}^{2}\right)}=1$ in (4.13).

Remark 4.1. As immediately follows from (4.13), a vector field $z: \Omega \rightarrow[-1,1]$ can be formally identified with the quotient $\frac{D u}{|D u|}$ provided $|D u|$ is nonzero and well defined at a given point $x \in \Omega$. However, due to the Azellotti's theory of pairing, the correct interpretation of the quotient $\frac{D u}{|D u|}$ can be made through the equality $(z, D u)_{\mathbb{R}^{2}}=|D u|$, where the field $z \in L_{2, \text { div }}^{\infty}\left(\Omega ; \mathbb{R}^{2}\right)$ is such that $\|z\|_{L^{\infty}\left(\Omega ; \mathbb{R}^{2}\right)} \leqslant 1$ (see [13] for the details).

Proof. We set $M^{*}$ is the set of functions $\xi \in L^{2}(\Omega)$ such that $\xi=-\operatorname{div} z$ for some $z \in L_{2, \text { div }}^{\infty}\left(\Omega ; \mathbb{R}^{2}\right)$, and $\|z\|_{L^{\infty}\left(\Omega ; \mathbb{R}^{2}\right)} \leqslant 1$ with $\operatorname{Tr}(z, \partial \Omega)=0$. In view of definition of the trace of the normal component of $z$ on $\partial \Omega$, this set is nonempty and well defined. Let us show that $M^{*}$ is closed with respect to the norm convergence in $L^{2}(\Omega)$. Let $\left\{\xi_{k}\right\}_{k \in \mathbb{N}} \subset M^{*}$ be a sequence such that $\xi_{k} \rightarrow \xi$ in $L^{2}(\Omega)$. Let $\left\{z_{k} \in L^{\infty}\left(\Omega ; \mathbb{R}^{2}\right)\right\}_{k \in \mathbb{N}}$ by their prototypes, i.e.,

$$
z_{k} \in L_{2, \text { div }}^{\infty}\left(\Omega ; \mathbb{R}^{2}\right),\left\|z_{k}\right\|_{L^{\infty}\left(\Omega ; \mathbb{R}^{2}\right)} \leqslant 1, \operatorname{Tr}\left(z_{k}, \partial \Omega\right)=0, \text { and } \xi_{k}=-\operatorname{div} z_{k} .
$$

Then, for all test functions $\varphi \in C_{0}^{\infty}\left(\mathbb{R}^{2}\right)$ and each $k \in \mathbb{N}$, we have the equalities

$$
\int_{\Omega}\left(z_{k}, \nabla \varphi\right)_{\mathbb{R}^{2}} d x=\int_{\Omega} \varphi \xi_{k} d x
$$

Since the sequence $\left\{z_{k}\right\}_{k \in \mathbb{N}}$ is bounded in $L^{\infty}\left(\Omega ; \mathbb{N}^{2}\right)$, by Banach-Alaoglu theorem, there exists a vector field $z \in L^{\infty}\left(\Omega ; \mathbb{N}^{2}\right)$ such that, up to a subsequence, we have 
the convergence $z_{k} \stackrel{*}{\rightarrow} z$ in $L^{\infty}\left(\Omega ; \mathbb{N}^{2}\right)$ as $k \rightarrow \infty$. Taking this fact into account and passing to the limit in (4.15) (for the chosen subsequence), we get

$$
(z, \nabla \varphi)_{\mathbb{R}^{2}} d x=\int_{\Omega} \varphi \xi d x, \quad \forall \varphi \in C_{0}^{\infty}\left(\mathbb{R}^{2}\right),
$$

i.e., in view of Lemma 2.1, we can deduce that

$$
\xi=-\operatorname{div} z \in L^{2}(\Omega) \quad \text { and } \quad\langle\operatorname{Tr}(z, \partial \Omega), \varphi\rangle=0
$$

$\forall \varphi \in C_{0}^{\infty}\left(\mathbb{R}^{2}\right)$. Making use of the lower semicontinuity of $L^{\infty}$-norm with respect to the weak ${ }^{*}$ convergence $\|z\|_{L^{\infty}\left(\Omega ; \mathbb{R}^{2}\right)} \leqslant \liminf _{k \rightarrow \infty}\left\|z_{k}\right\|_{L^{\infty}\left(\Omega ; \mathbb{R}^{2}\right)} \leqslant 1$, we finally obtain $\xi \in M^{*}$. Thus, $M^{*}$ is a closed subset of $L^{2}(\Omega)$.

Let $I_{M^{*}}$ be the indicator function of $M^{*}$. Then the Young-Fenchel transform of $I_{M^{*}}$ is the function on $L^{2}(\Omega)$ defined by

$$
I_{M^{*}}^{*}(v)=\sup _{\xi \in L^{2}(\Omega)}\left\{(\xi, v)_{L^{2}(\Omega)}-I_{M^{*}}(\xi)\right\}=\sup _{\xi \in M^{*}}\left\{(\xi, v)_{L^{2}(\Omega)}\right\} \quad \forall v \in L^{2}(\Omega) .
$$

Fixing arbitrary $\xi \in M^{*}$ and $v \in L^{2}(\Omega)$, we see that

$$
\begin{aligned}
& (\xi, v)_{L^{2}(\Omega)}=\int_{\Omega} \xi v d x \stackrel{\text { by definition of } M^{*}}{=}-\int_{\Omega} v \operatorname{div} z d x \\
& =\int_{\Omega} d(z, D v) \stackrel{\text { by }}{\stackrel{(2.12)}{\leqslant}}\|z\|_{L^{\infty}\left(\Omega ; \mathbb{R}^{2}\right)} \int_{\widehat{\Omega}} d|D v| \\
& \leqslant \int_{\Omega} d|D v|=E_{1}(v) \text {. }
\end{aligned}
$$

Hence,

$$
I_{M^{*}}^{*}(v) \leqslant E_{1}(v) \quad \forall v \in L^{2}(\Omega) .
$$

On the other hand, by definition of the total variation of $v$ in $\Omega$, we get

$$
E_{1}(v)=+\infty \quad \text { provided } \quad v \in L^{2}(\Omega) \backslash B V(\Omega)
$$

and

$$
\begin{aligned}
& E_{1}(v)=\int_{\Omega} d|D v|=\left.\sup \int_{\Omega} v \operatorname{div} z d x\right|_{\substack{z \in C_{0}^{\infty}\left(\Omega ; \mathbb{R}^{2}\right),\|z\|_{L^{\infty}\left(\Omega ; \mathbb{R}^{2}\right)} \leqslant 1}} \\
& \leqslant\left.\sup \int_{\Omega} v \operatorname{div} z d x\right|_{\substack{z \in L_{0}^{\infty}\left(\Omega ; \mathbb{R}^{2}\right),\|z\|_{L^{\infty}\left(\Omega ; \mathbb{R}^{2}\right)} \leqslant 1, \operatorname{div} z \in L^{2}(\Omega), \quad \text { with } \operatorname{Tr}(z, \partial \Omega)=0}} \\
& =\left.\sup \left\{-\int_{\Omega} v \operatorname{div} z d x\right\}\right|_{z \in L_{0}^{\infty}\left(\Omega ; \mathbb{R}^{2}\right),\|z\|_{L^{\infty}\left(\Omega ; \mathbb{R}^{2}\right)} \leqslant 1,} \\
& =\sup \left\{\int_{\Omega} v \xi d x \mid \xi \in M^{*}\right\}=I_{M^{*}}^{*}(v), \forall v \in B V(\Omega) .
\end{aligned}
$$


As a result, we deduce from (4.17) that

$$
I_{M^{*}}^{*}(v)=E_{1}(v), \quad \forall v \in L^{2}(\Omega) .
$$

Taking into account the fact that $M^{*}$ is a closed and convex subset of $L^{2}(\Omega)$, it follows that its indicator function $I_{M^{*}}$ is convex and lower semicontinuous. Therefore, by the well known results of convex analysis (see [15, Proposition 3.1]), we have

$$
I_{M^{*}}=\left(I_{M^{*}}^{*}\right)^{*}=E_{1}^{*} .
$$

Consequently, $\xi \in \partial E_{1}(v)$ if and only if

$$
(\xi, v)_{L^{2}(\Omega)}=E_{1}(v)+E^{*}(\xi)=E_{1}(v)+I_{M^{*}}(\xi) .
$$

Thus, from this we immediately conclude that

$$
\xi \in \partial E_{1}(v) \text { if and only if } E_{1}(v)=(\xi, v)_{L^{2}(\Omega)}, \forall \xi \in M^{*} .
$$

It remains to notice that the last equality together with estimate (4.16) implies the relation

$$
(\xi, v)_{L^{2}(\Omega)}=\|z\|_{L^{\infty}\left(\Omega ; \mathbb{R}^{2}\right)} \int_{\widehat{\Omega}} d|D v|=E_{1}(v) .
$$

Hence, $\|z\|_{L^{\infty}\left(\Omega ; \mathbb{R}^{2}\right)}=1$ provided $E_{1}(v)<+\infty$.

We are now in a position to derive the necessary conditions for a unique minimizer $u_{i}^{o p t} \in \Xi \subset B V(\Omega)$ of constrained minimization problem (3.9)-(3.10). Since $u_{i}^{\text {opt }}$ is also a minimizer of the modified problem (4.6) and the functionals $\widehat{J}_{i}: L^{2}(\Omega) \rightarrow \mathbb{R}$ and $G: L^{2}(\Omega) \rightarrow \mathbb{R}$ are convex, a necessary conditions should employ the convex subdifferentials $\partial \widehat{J}_{i}\left(u_{i}^{o p t}\right)$ and $\partial G\left(u_{i}^{o p t}\right)$. As a result, utilizing Proposition 6.3 in [21], we arrive at the following result.

Theorem 4.1. Assume that, for given $i \in\{1, \ldots, M-1\}, \lambda_{1} \geqslant 0, \lambda_{2}>0$, $u_{i, d} \in L^{2}(\Omega)$, and $v_{i, d} \in L^{2}(\Omega)$, and condition (ii) holds true. Let $u_{i}^{\text {opt }} \in \Xi$ be a minimizer of constrained minimization problem (3.9)-(3.10). Then there is a vector-valued field $z \in L^{\infty}\left(\Omega ; \mathbb{R}^{2}\right)$ with $\operatorname{div} z \in L^{2}(\Omega)$ such that

$$
\begin{gathered}
-\operatorname{div} z+2 \lambda_{1}(-\Delta)^{-1}\left(u_{i}^{o p t}-u_{i, d}\right) \\
+2 \lambda_{2} B_{i}^{*}\left(B_{i}\left(u_{i}^{o p t}\right)-v_{i, d}\right)=0 \quad \text { a.e. in } \Omega, \\
u_{i}^{\text {opt }}=\left|u_{i}^{\text {opt }}\right| \quad \text { a.e. in } \Omega, \\
\|z\|_{L^{\infty}\left(\Omega ; \mathbb{R}^{2}\right)}=1, \int_{\Omega} d\left|D u_{i}^{\text {opt }}\right|=-\int_{\Omega} u_{i}^{\text {opt }} \operatorname{div} z d x, \\
\operatorname{Tr}(z, \partial \Omega)=0 .
\end{gathered}
$$


Remark 4.2. If $\left|D u_{i}^{\text {opt }}\right|$ is nonzero and well-defined, a vector field $z: \Omega \rightarrow B_{1}(0)$ with the above mentioned properties can be identified with $\frac{D u_{i}^{o p t}}{\left|D u_{i}^{o p t}\right|}$ and, therefore, relations (4.20)-(4.23) can be interpreted as the following Neumann boundary value problem for the 1-Laplace operator

$$
\begin{aligned}
&-\operatorname{div}\left(\frac{D u_{i}^{o p t}}{\left|D u_{i}^{o p t}\right|}\right)=-2 \lambda_{1}(-\Delta)^{-1}\left(u_{i}^{o p t}-u_{i, d}\right) \\
&-2 \lambda_{2} B_{i}^{*}\left(B_{i}\left(u_{i}^{o p t}\right)-v_{i, d}\right) \text { in } \Omega, \\
&\left(\frac{D u_{i}^{o p t}}{\left|D u_{i}^{o p t}\right|}, \nu\right)_{\mathbb{R}^{2}}=0 \quad \text { on } \partial \Omega, \\
& u_{i}^{o p t} \geqslant 0 \quad \text { a.e. in } \Omega .
\end{aligned}
$$

Proof. Let $u_{i}^{o p t} \in \Xi \subset B V(\Omega)$ be a minimizer of (4.6). Then Proposition 3.1 implies that $\widehat{J}_{i}\left(u_{i}^{o p t}\right)<+\infty$ and $G\left(u_{i}^{o p t}\right)=0$. Observing that the minimizer $u_{i}^{o p t}$ of (3.9)-(3.10) is also a minimizer of the modified problem (4.6) and it is an unconstrained minimizer of the function $\widehat{J}_{i}+I_{G}$ on $X=L^{2}(\Omega)$ with the indicator function

$$
I_{G}(v):= \begin{cases}0 & \text { if } G(v)=0 \\ \infty & \text { otherwise }\end{cases}
$$

the derivation of a non-smooth Lagrange multiplier rule for problem (4.6) can be done as in [21, Proposition 6.3]. As a result, using the sum rule for subdifferential of convex functionals saying that

$$
\partial\left(\widehat{J}_{i}+I_{G}\right)(u)=\partial E_{1}(u)+\partial E_{2}(u)+\partial E_{3}(u)+\partial I_{G}(u),
$$

we deduce the existence of elements

$$
u_{1}^{*} \in \partial E_{1}\left(u_{i}^{o p t}\right), u_{2}^{*} \in \partial E_{2}\left(u_{i}^{o p t}\right), u_{3}^{*} \in \partial E_{3}\left(u_{i}^{o p t}\right), \text { and } u_{4}^{*} \in \partial G\left(u_{i}^{o p t}\right)
$$

such that

$$
u_{1}^{*}+u_{2}^{*}+u_{3}^{*}+t u_{4}^{*}=0, \quad \forall t>0 .
$$

Hence, in view of Propositions 4.1, 4.2, 4.3, and 4.4 (see also equality (4.19)), we arrive at the announced relations (4.20)-(4.23).

\subsection{Optimality Conditions for Segmentation Problem (3.7)}

In order to derive the necessary conditions of optimality in optimal segmentation problem (3.7), we make use of the so-called boundary variation method (for the details of this approach, we refer to [8, Section 1.2]). Let $E^{o p t} \subseteq \Omega$ be an essential optimal solution to variational problem (3.7). Then

$$
0<P\left(E^{o p t} ; \Omega\right)=\left|D \chi_{E^{o p t}}\right|(\Omega)=\int_{\Omega} d\left|D \chi_{E^{o p t}}\right|<\infty
$$


and $\chi_{E^{\text {opt }}} \in S B V(\Omega)$. Without loss of generality, we assume that the set $E^{\text {opt }} \subseteq \Omega$ has a Lipschitz boundary and, therefore, we can suppose that

$$
\int_{\Omega} d\left|D \chi_{E^{o p t}}\right|=\mathcal{H}^{1}\left(\Omega \cap \partial^{*} E^{o p t}\right)
$$

and $D \chi_{E^{o p t}}=-\nu_{E^{o p t}}(x) \mathcal{H}^{1}\left\llcorner\partial^{*} E^{o p t}\right.$, where $\nu_{E^{o p t}}(x)$ stands for the exterior unit normal vector to $E^{o p t}$ at $x \in \partial^{*} E^{o p t}$.

Following the boundary variation method, we assume that $E^{\text {opt }}$ is an open subset of $\Omega$ and its boundary $\partial E^{o p t}$ is regular in the following sense: there exists a $\delta>0$, a finite number of points $\left\{\xi_{k}\right\}_{k=1}^{m} \subset \partial E^{o p t}$, and a collection of $C^{0,1}$ functions $\left\{\phi^{k}: \mathbb{R} \rightarrow \mathbb{R}\right\}_{k=1}^{m}$ such that

$$
\begin{aligned}
\Omega \cup \partial \Omega & \subset \bigcup_{k=1}^{m} \Pi_{2}\left(\xi^{k}\right), \\
\Pi_{2}\left(\xi^{k}\right) & =\left\{x \in \mathbb{R}^{2}:\left|x_{i}-\xi_{i}^{k}\right|<\delta, i=1,2\right\}, \\
E^{o p t} \cap \Pi_{2}\left(\xi^{k}\right) & =\left\{x \in \Pi_{2}\left(\xi^{k}\right): x_{2}<\phi_{k}\left(x_{1}\right)\right\}, \\
\left(\Omega \backslash \overline{E^{o p t}}\right) \cap \Pi_{2}\left(\xi^{k}\right) & =\left\{x \in \Pi_{2}\left(\xi^{k}\right): x_{2}>\phi_{k}\left(x_{1}\right)\right\}, \\
\partial E^{o p t} \cap \Pi_{2}\left(\xi^{k}\right) & =\left\{x \in \Pi_{2}\left(\xi^{k}\right): x_{2}=\phi_{k}\left(x_{1}\right)\right\} .
\end{aligned}
$$

Actually, as it is mentioned in [8], the regularity of $\partial E^{o p t}$ does not need to be assumed as a hypothesis but it is a consequence of some suitable conditions on the class of feasible solutions to the problem (3.7). Since this is a quite delicate matter which goes under the name of regularity theory, we do not drill down into this field and we refer the interesting reader to the various books available in the literature (see, for instance, references $[18,23]$ ). Thus, in view of the above given assumptions, for $\mathcal{H}^{1}$-almost all $x_{0} \in \partial E^{\text {opt }}$ the boundary $\partial E^{o p t}$ near a given point $x_{0} \in \partial E^{o p t}$ can be written as the graph of some function $\phi(x)$, where $x$ varies in an open subset $\omega$ of $\mathbb{R}$. Namely, for a fixed $x_{0} \in \partial E^{\text {opt }}$, there exists $\widehat{k} \in\{1, \ldots, m\}$ such that representations (4.29)-(4.31) remain valid for $k=\widehat{k}$. So, we can suppose that $\omega=\left\{x_{1} \in \mathbb{R}:\left|x_{1}-\xi_{1}^{\widehat{k}}\right|<\delta\right\}$. Since, without loss of generality, we can suppose that

$$
J_{0}\left(E^{o p t}\right)=\sum_{k=1}^{m} I_{k}\left(\phi_{k}\right),
$$

where

$$
\begin{aligned}
I_{k}\left(\phi_{k}\right)= & \lambda_{3} \int_{\Pi_{2}\left(\xi^{k}\right)} d\left|D \chi_{E^{o p t}}\right|+\int_{\Pi_{2}\left(\xi^{k}\right)}\left|V\left(\mathbf{u}^{o p t}\right)-c_{1}\right|^{r} \chi_{E^{o p t}} d x \\
& +\int_{\Pi_{2}\left(\xi^{k}\right)}\left|V\left(\mathbf{u}^{o p t}\right)-c_{2}\right|^{r}\left(1-\chi_{E^{o p t}}\right) d x,
\end{aligned}
$$


it follows that the corresponding part of the energy functional $J_{0}$ (in the region of $x_{0} \in \partial E^{o p t}$ ) can be represented in the Cartesian form as follows

$$
\begin{aligned}
I_{\widehat{k}}\left(\phi_{\widehat{k}}\right)= & \lambda_{3} \int_{\omega} \sqrt{1+\left|D \phi_{\widehat{k}}\left(x_{1}\right)\right|^{2}} d x_{1}+\int_{\omega} \int_{\phi_{\widehat{k}}\left(x_{1}\right)}^{\xi_{2}^{\widehat{k}}+\delta}\left|V\left(\mathbf{u}^{o p t}\right)-c_{1}\right|^{r} d x_{2} d x_{1} \\
& +\int_{\omega} \int_{\xi_{2}^{\widehat{k}}-\delta}^{\phi_{\widehat{k}}\left(x_{1}\right)}\left|V\left(\mathbf{u}^{o p t}\right)-c_{2}\right|^{r} d x_{2} d x_{1},
\end{aligned}
$$

where the symbol $D$ stands for the differentiation operator $d / d x_{1}$. We emphasize that because of the Lipschitz property of functions $\phi^{k}: \mathbb{R} \rightarrow \mathbb{R}$, they are differentiable for almost all $x_{1} \in \omega$.

Following the boundary variation method, we perturb of $\partial E^{o p t}$, and hence $\phi_{\widehat{k}}\left(x_{1}\right)$, by taking a comparison function of the form $\phi_{\widehat{k}}\left(x_{1}\right)+\varepsilon \psi\left(x_{1}\right)$, where $\varepsilon>0$ is a small parameter, and $\psi: \omega \rightarrow \mathbb{R}$ is a smooth function with support in $\omega$. Since $E^{\text {opt }}$ is a minimizer for the problem (3.7), this leads us to the inequality

$$
I_{\widehat{k}}\left(\phi_{\widehat{k}}+\varepsilon \psi\right) \geqslant I_{\widehat{k}}\left(\phi_{\widehat{k}}\right), \quad \forall \varepsilon>0 .
$$

For our further analysis, we make use of the following representations

$$
\begin{aligned}
& \int_{\omega} \sqrt{1+\left|D \phi_{\widehat{k}}+\varepsilon D \psi\right|^{2}} d x_{1}= \int_{\omega} \sqrt{1+\left|D \phi_{\widehat{k}}\right|^{2}} d x_{1} \\
&+\varepsilon \int_{\omega} \frac{D \phi_{\widehat{k}} D \psi}{\sqrt{1+\left|D \phi_{\widehat{k}}\right|^{2}}} d x_{1}+o(\varepsilon), \\
& \int_{\omega} \int_{\phi_{\widehat{k}}\left(x_{1}\right)+\varepsilon \psi\left(x_{1}\right)}^{\xi_{2}^{\widehat{k}}+\delta}\left|V\left(\mathbf{u}^{o p t}\right)-c_{1}\right|^{r} d x_{2} d x_{1}=\int_{\omega} \int_{\phi_{\widehat{k}}\left(x_{1}\right)}^{\xi_{2}^{\widehat{k}}+\delta}\left|V\left(\mathbf{u}^{o p t}\right)-c_{1}\right|^{r} d x_{2} d x_{1} \\
&-\varepsilon \int_{\omega} \psi\left(x_{1}\right)\left[\frac{1}{\varepsilon \psi\left(x_{1}\right)} \Phi_{1}\left(x_{1}\right)\right] d x_{1}, \\
& \int_{\omega} \int_{\xi_{2}^{\widehat{k}}-\delta}^{\phi_{\widehat{k}}\left(x_{1}\right)+\varepsilon \psi\left(x_{1}\right)}\left|V\left(\mathbf{u}^{o p t}\right)-c_{2}\right|^{r} d x_{2} d x_{1}=\int_{\omega} \int_{\xi_{2}^{\widehat{k}}-\delta}^{\phi_{\widehat{k}}\left(x_{1}\right)}\left|V\left(\mathbf{u}^{o p t}\right)-c_{2}\right|^{r} d x_{2} d x_{1} \\
&+\varepsilon \int_{\omega} \psi\left(x_{1}\right)\left[\frac{1}{\varepsilon \psi\left(x_{1}\right)} \Phi_{2}\left(x_{1}\right)\right] d x_{1},
\end{aligned}
$$

where

$$
\begin{aligned}
& \Phi_{1}\left(x_{1}\right)=\int_{\phi_{\widehat{k}}\left(x_{1}\right)}^{\phi_{\widehat{k}}\left(x_{1}\right)+\varepsilon \psi\left(x_{1}\right)}\left|V\left(\mathbf{u}^{o p t}\right)-c_{1}\right|^{r} d x_{2}, \\
& \Phi_{2}\left(x_{1}\right)=\int_{\phi_{\widehat{k}}\left(x_{1}\right)}^{\phi_{\widehat{k}}\left(x_{1}\right)+\varepsilon \psi\left(x_{1}\right)}\left|V\left(\mathbf{u}^{o p t}\right)-c_{2}\right|^{r} d x_{2} .
\end{aligned}
$$

In view of the initial assumptions, we see that

$$
\left|V\left(\mathbf{u}^{\text {opt }}\right)-c_{i}\right|^{r} \in L^{\infty}(\Omega), \quad i=1,2 .
$$


Hence, by Lebesgue differentiation theorem, almost every point $x \in \Omega$ is a Lebesgue point of $\left|V\left(\mathbf{u}^{o p t}\right)-c_{1}\right|^{r}$ and $\left|V\left(\mathbf{u}^{o p t}\right)-c_{2}\right|^{r}$. Therefore, setting

$$
\begin{aligned}
& \left|V\left(\mathbf{u}^{o p t}\left(x_{1}, \phi_{\widehat{k}}\left(x_{1}\right)\right)\right)-c_{i}\right|^{r} \\
& :=\lim _{\varepsilon \rightarrow 0+0} \frac{1}{\varepsilon \psi\left(x_{1}\right)} \int_{\phi_{\widehat{k}}\left(x_{1}\right)}^{\phi_{\widehat{k}}\left(x_{1}\right)+\varepsilon \psi\left(x_{1}\right)}\left|V\left(\mathbf{u}^{o p t}\right)-c_{i}\right|^{r} d x_{2}, i=1,2,
\end{aligned}
$$

we deduce from (4.32) that

$$
\begin{aligned}
\lambda_{3} \int_{\omega} \frac{D \phi_{\widehat{k}} D \psi}{\sqrt{1+\left|D \phi_{\widehat{k}}\right|^{2}}} d x_{1} & +\int_{\omega}\left|V\left(\mathbf{u}^{\text {opt }}\left(x_{1}, \phi_{\widehat{k}}\left(x_{1}\right)\right)\right)-c_{2}\right|^{r} \psi\left(x_{1}\right) d x_{1} \\
& -\int_{\omega}\left|V\left(\mathbf{u}^{\text {opt }}\left(x_{1}, \phi_{\widehat{k}}\left(x_{1}\right)\right)\right)-c_{1}\right|^{r} \psi\left(x_{1}\right) d x_{1} \geqslant 0 .
\end{aligned}
$$

Integrating by parts and taking into account that $\psi \in C_{0}^{\infty}(\omega)$, we obtain

$$
\begin{aligned}
-\lambda_{3} \int_{\omega} \frac{d}{d x_{1}} & \left(\frac{D \phi_{\widehat{k}}}{\sqrt{1+\left|D \phi_{\widehat{k}}\right|^{2}}}\right) \psi\left(x_{1}\right) d x_{1} \\
\geqslant & \int_{\omega}\left|V\left(\mathbf{u}^{o p t}\left(x_{1}, \phi_{\widehat{k}}\left(x_{1}\right)\right)\right)-c_{1}\right|^{r} \psi\left(x_{1}\right) d x_{1} \\
& -\int_{\omega}\left|V\left(\mathbf{u}^{o p t}\left(x_{1}, \phi_{\widehat{k}}\left(x_{1}\right)\right)\right)-c_{2}\right|^{r} \psi\left(x_{1}\right) d x_{1} .
\end{aligned}
$$

Since $\psi \in C_{0}^{\infty}(\omega)$ is an arbitrary function, we finally deduce that $\phi_{\widehat{k}} \in C^{0,1}(\omega)$ must satisfy the following differential equation

$$
\begin{aligned}
-\lambda_{3} \frac{d}{d x_{1}}\left(\frac{D \phi_{\widehat{k}}}{\sqrt{1+\left|D \phi_{\widehat{k}}\right|^{2}}}\right)= & \left|V\left(\mathbf{u}^{\text {opt }}\left(x_{1}, \phi_{\widehat{k}}\left(x_{1}\right)\right)\right)-c_{1}\right|^{r} \\
& -\left|V\left(\mathbf{u}^{o p t}\left(x_{1}, \phi_{\widehat{k}}\left(x_{1}\right)\right)\right)-c_{2}\right|^{r} \quad \text { in } \omega,
\end{aligned}
$$

where the term

$$
-\frac{d}{d x_{1}}\left(\frac{D \phi_{\widehat{k}}}{\sqrt{1+\left|D \phi_{\widehat{k}}\right|^{2}}}\right)
$$

represent the mean curvature of $\partial E^{o p t}$ written in Cartesian coordinates. Thus, we have arrived at the following necessary conditions of optimality for a regular solution $E^{\text {opt }} \subset \Omega$ of segmentation problem (3.7): the mean curvature of $\partial E^{o p t} \cap$ $\Pi_{2}\left(\xi^{k}\right)$ is locally equal to

$$
\lambda_{3}^{-1}\left[\left|V\left(\mathbf{u}^{o p t}\left(x_{1}, \phi_{\widehat{k}}\left(x_{1}\right)\right)\right)-c_{1}\right|^{r}-\left|V\left(\mathbf{u}^{o p t}\left(x_{1}, \phi_{\widehat{k}}\left(x_{1}\right)\right)\right)-c_{2}\right|^{r}\right]
$$

for each $k=1, \ldots, m$. 


\section{Application to the Satellite Image Processing Problem}

It is well known that the satellite images can be considered as a source of knowledge used for monitoring and evaluating the Earth's vegetative cover in order to estimate the amount of vegetation, its biomass, percent of coverage, and provide the distinctions between bare soils and vegetation zone. One of the standard ways enabling to get such information is determination and timedependent analysis of the so-called vegetation indices. The most commonly used vegetation index (VI) is the so-called slope-based Infrared Percentage Vegetation Index (IPVI),

$$
\mathrm{IPVI}:=\frac{u_{2, d}}{u_{1, d}+u_{2, d}}, \quad 0 \leqslant \mathrm{IPVI} \leqslant 1
$$

where $u_{i, d}=u_{i, d}\left(x_{1}, x_{2}\right), i=1,2$, with $\left(x_{1}, x_{2}\right) \in \Omega$ are functions of two variables representing the intensity of red $(R e d)$ and near-infrared $(N I R)$ reflectance of some region $\Omega$ of $\mathbb{R}^{2}$, respectively.

Let $\Omega=\left(a_{1}, b_{1}\right) \times\left(a_{2}, b_{2}\right)$ be a rectangle domain in $\mathbb{R}^{2}$. We associate with this domain the mapping

$$
F: \Omega \rightarrow\left[\begin{array}{l}
u_{1, d} \\
u_{2, d}
\end{array}\right] \in \mathbb{R}^{2}
$$

as a two-bands satellite image representing the intensity of red reflectance (Red) $u_{1, d}$ and near-infrared reflectance $(N I R) u_{2, d}$ of the region $\Omega$, respectively. The problem, which is suggested by application to remote sensing satellite image processing, consists in computing a decomposition $\Omega=\Omega_{1} \cup \Omega_{2} \cup \cdots \cup \Omega_{K}$ such that the IPVI-characteristic (5.1) varies slowly within each $\Omega_{j}$, and this characteristic varies discontinuously and/or rapidly across most of the boundary between different $\Omega_{j}$. Let us show that this problem can be considered as a particular case of the coupled optimization problem (3.2)-(3.7).

With that in mind we notice that the observed imaging data $u_{i, d}, i=1,2$, typically suffer from noise and blurs. So, it is reasonably to consider the deblurring and de-noising problem as the first step of preprocessing for the mapping $F: \Omega \rightarrow \mathbb{R}^{2}$ in order to recover the original high resolution image which is unknown a priori but it must be, in some sense, as close as possible to the observed data $u_{i, d}, i=1,2$. The core idea of our approach to the de-noising and de-blurring problem of two-band satellite images is to use the total variation as a regularizing term in the following variational problems

$$
\begin{aligned}
J_{j}(u)=\int_{\Omega} d|D u| & +\lambda_{1}\left\|u-u_{j, d}\right\|_{H^{-1}(\Omega)}^{2} \\
& +\lambda_{2}\left\|B_{j}(u)-u_{j, d}\right\|_{L^{2}(\Omega)}^{2} \rightarrow \inf _{u \in \Xi}, j=1,2 .
\end{aligned}
$$

Here, $\lambda_{i}>0$ are the tuning parameters,

$$
\Xi=\{u \in B V(\Omega): u(x) \geqslant 0 \text { a.e. in } \Omega\}
$$


is the set of feasible solutions, the operators $B_{1}, B_{2}: L^{2}(\Omega) \rightarrow L^{2}(\Omega)$ are defined as follows

$$
B_{i}(u)=\frac{1}{2 \pi \sigma_{i}^{2}} \int_{\Omega} u(x-y) e^{-|y|^{2} / 2 \sigma_{i}^{2}} d y, \quad i=1,2,
$$

and the expressions $\left\|u-u_{j, d}\right\|_{H^{-1}(\Omega)}^{2}$ and $\left\|B_{1}(u)-u_{j, d}\right\|_{L^{2}(\Omega)}^{2}$ stand for the denoising and de-blurring terms, respectively.

As follows from Proposition 3.1, for given $\lambda_{1} \geqslant 0, \lambda_{2}>0$, and $u_{i, d} \in L^{2}(\Omega)$, $i=1,2$, the constrained minimization problems (5.3) admit unique solutions $u_{1}^{o p t}$ and $u_{2}^{o p t}$, respectively. Further, we define the so-called selective smoothing operators $\left\{V_{i}: B V(\Omega) \rightarrow B V(\Omega)\right\}_{i=1}^{2}$ as follows: for the de-blurred and de-noised images $u_{i}^{\text {opt }} \in B V(\Omega), i=1,2$, we set up $V_{i}\left(u_{i}^{\text {opt }}\right)$ as a steady-state solution of the following initial-boundary value problem with anisotropic diffusion operator

$$
\begin{aligned}
\frac{\partial S_{i}}{\partial t} & =g_{i}\left(\left|G_{i} * D S_{i}\right|\right)\left|D S_{i}\right| \operatorname{div}\left(\frac{D S_{i}}{\left|D S_{i}\right|}\right) \quad \text { in }(0, \infty) \times \Omega, \\
\partial_{\nu} S_{i} & =0 \quad \text { on }(0, \infty) \times \partial \Omega, \\
S_{i}(0, \cdot) & =T\left[u_{i}^{o p t}\right](\cdot) \quad \text { a.e. in } \Omega .
\end{aligned}
$$

Here, $G_{i}$ is the Gauss smoothing kernel, $T(u):=\int_{\Omega} T(x-y) u(y) d y \in W^{1, \infty}(\Omega)$ is the Steklov smoothing operator with positive compactly supported smooth function $T: \mathbb{R}^{2} \rightarrow \mathbb{R}$ such that

$$
T \in C_{0}^{\infty}\left(\mathbb{R}^{2}\right), \int_{\mathbb{R}^{2}} T(x) d x=1, \text { and } T(x)=T(-x),
$$

and $g_{i}(\cdot)$ is the edge stopping function that we define as follows

$$
g_{i}(s)=\frac{1}{1+k_{i} s^{2}}
$$

It should be noted that the system (5.5)-(5.7) belongs to the class of well-posed problems (existence and uniqueness were proven in [10], for the details we refer to [2, Section 3]). As a result, it can be showed that the above mentioned operators $V_{i}$ are sequentially continuous. Thus, the optimal satellite image segmentation problem via piecewise smooth approximation of Infrared Percentage Vegetation Index (5.1) can be stated as follows: Find a nonempty subset $E^{o p t} \subset \Omega$ such that:

$$
\begin{gathered}
E^{\text {opt }} \in \Xi_{0}=\{E \subseteq \Omega: P(E, \Omega)<+\infty\}, \\
\mathcal{L}^{2}\left(E^{\text {opt }} \cap B(x)\right)>0 \text { for any } x \in E^{\text {opt }} \text { and any ball } B(x) \subset \Omega, \text { and } \\
J_{0}\left(E^{o p t}\right)=\inf _{E \in \Xi_{0}} J_{0}(E),
\end{gathered}
$$


where

$$
\begin{aligned}
J_{0}(E)= & \int_{\Omega} d\left|D \chi_{E}\right|+\int_{\Omega}\left|V\left(\mathbf{u}^{o p t}\right)-c_{1}\right|^{2} \chi_{E} d x \\
& +\int_{\Omega}\left|V\left(\mathbf{u}^{o p t}\right)-c_{2}\right|^{2}\left(1-\chi_{E}\right) d x, \\
V\left(\mathbf{u}^{o p t}\right)= & \frac{V_{2}\left(u_{2 R}^{o p t}\right)}{V_{1}\left(u_{1}^{o p t}\right)+V_{2}\left(u_{2}^{o p t}\right)} \text { a.e. in } \Omega,
\end{aligned}
$$

values $c_{1}$ and $c_{2}$ are defined by the rules (3.5)-(3.6) and $V_{i}\left(u_{i}^{\text {opt }}\right), i=1,2$, are steady-state solutions of the problem (5.5)-(5.7).

It remains to notice that, in view of Remark 3.2 and Theorem 3.1, this segmentation problem admits at least one optimal partition $\Omega=E^{\text {opt }} \cup\left(\Omega \backslash E^{\text {opt }}\right)$ that can be characterized by necessary optimality conditions (4.33).

\subsection{Results of Numerical Simulation}

We illustrate here the accuracy and efficiency of the proposed algorithm by numerical experiences with images that have been delivered by satellite Sentinel2. From technical point of view, Sentinel-2 carries a multispectral imager with a swath of $290 \mathrm{~km}$, and delivers high-resolution optical images for land monitoring, emergency response and security services. The imager provides a versatile set of 13 spectral bands spanning from the visible and near infrared to the shortwave infrared, featuring four spectral bands at $10 \mathrm{~m}$, six bands at $20 \mathrm{~m}$ and three bands at $60 \mathrm{~m}$ spatial resolution.

In what follows, we associate with the original image (see Figure 5.1) the mapping (5.2), where the intensities of red and infrared reflectance $u_{1, d}, u_{2, d}$ are presented in Figures 5.3-5.4.

As follows from the pictures given in Figures 5.1-5.2 (see also the corresponding histogram of IPVI-characteristic for the original image), the observed data $u_{i, d}=u_{i, d}\left(x_{1}, x_{2}\right), i=1,2$, suffer from noise and blurs.

In accordance with algorithm that we propose in Section 5 , we consider the de-blurring and de-noising problem for $u_{i, d}=u_{i, d}\left(x_{1}, x_{2}\right), i=1,2$, as the first step of the image preprocessing. To do so, we solve the variational problems (5.3) with $\lambda_{1}=20$ and $\lambda_{2}=10$. Figures 5.5 and 5.6 contain the corresponding solutions $u_{1}^{o p t}$ and $u_{2}^{o p t}$ of these problems.

The next step is to solve the system (5.5)-(5.7) and find its steady-state solutions $V_{i}\left(u_{i}^{o p t}\right)$ for the corresponding deblurred images $u_{i}^{\text {opt }}$. Making use of the standard approach for the numerical simulation of the initial-boundary value problem (see, for instance, [32] for the details), we show that the chosen selective smoothing procedure transforms the original image and its IPVI-characteristic to their 'cartoon' versions as it is shown in Figures 5.7 and 5.8.

Results of optimal segmentation are presented on Figures 5.9-5.14. 


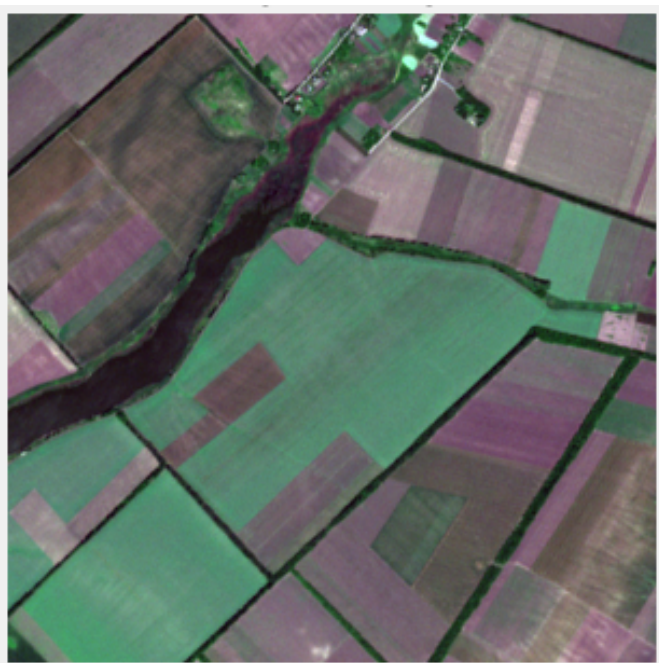

Fig. 5.1. Original Sentinel-2 image

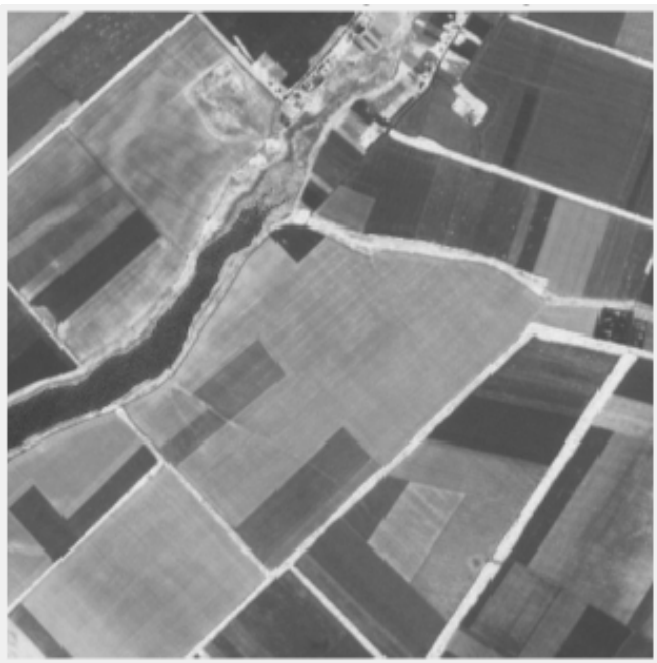

Fig. 5.2. IPVI for the original image Example the crop field and the corresponding IPVI-values rescaled to the range $[0,255]$
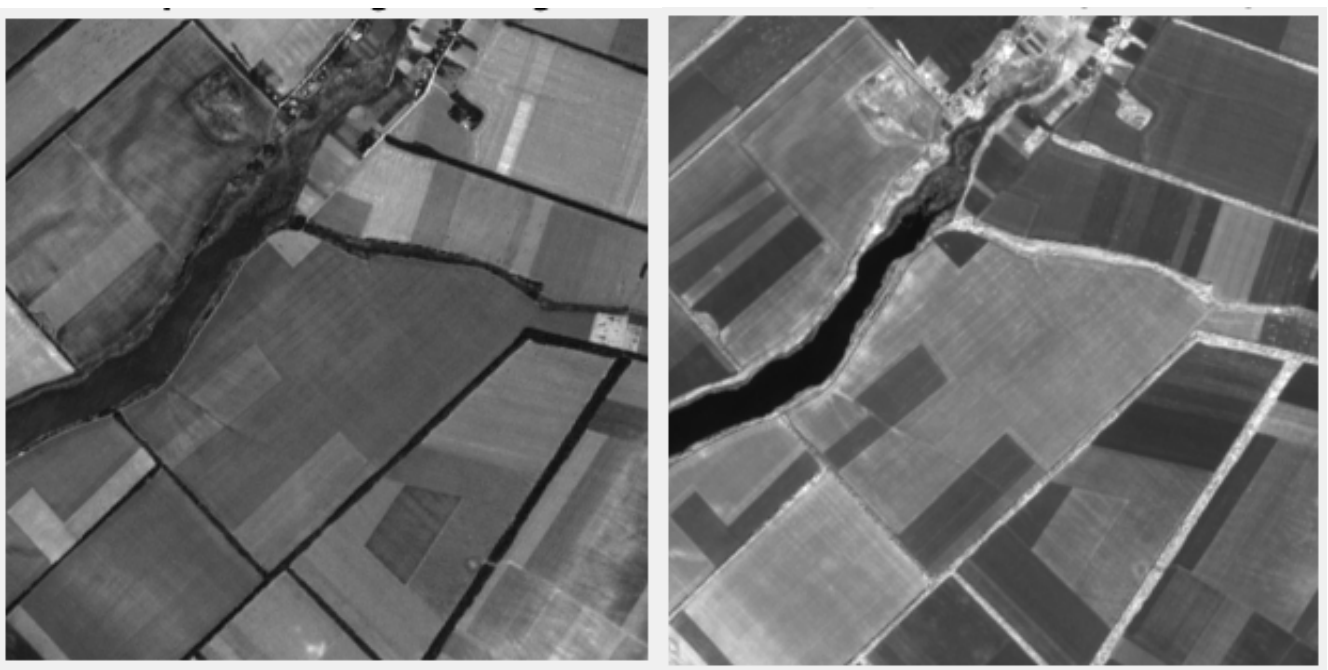

Fig. 5.3. Red band of the original image

Fig. 5.4. Infrared spectrum of the same image Red band and near-infrared region of the electromagnetic spectrum of the original image

\section{Conclusion}

In this paper, we have proposed a new setting for the optimal image segmentation problem which is based on the concept of piecewise smooth approximation of some selective target mappings. We have shown that the remote sensing satellite image segmentation problem, based on the analysis of the slope-based vegetation indices, is a particular case of the proposed setting. Focusing mainly on the rigor mathematical substantiation of the proposed approach, we discuss in details the 


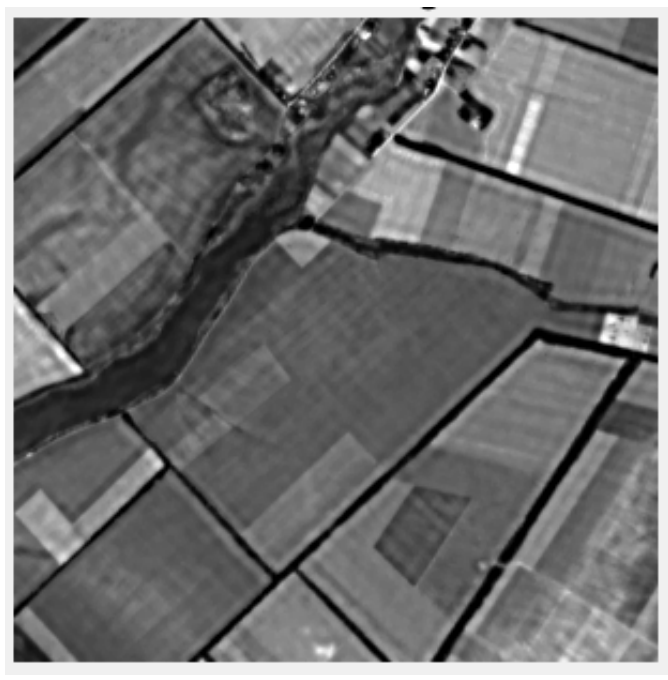

Fig. 5.5. $u_{1}^{o p t}$

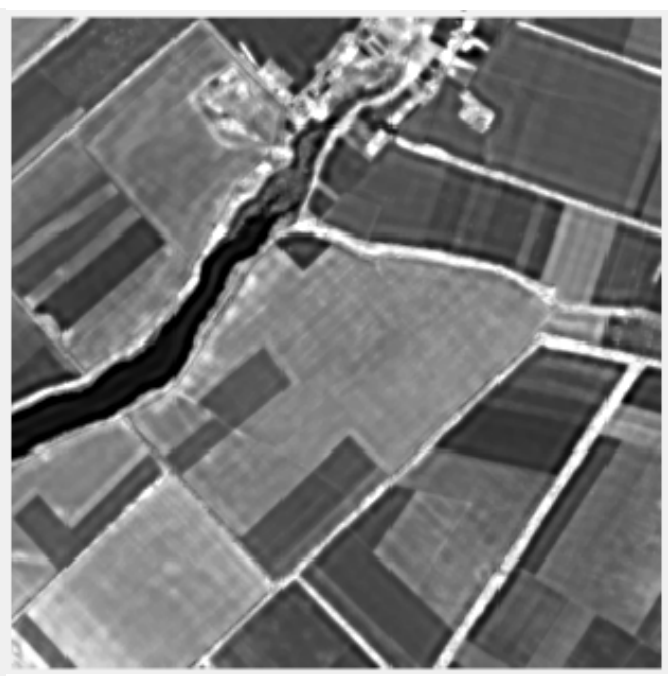

Fig. 5.6. $u_{2}^{o p t}$

Red band and near-infrared spectrum in high resolution after denoising and deblurring processing of the original image

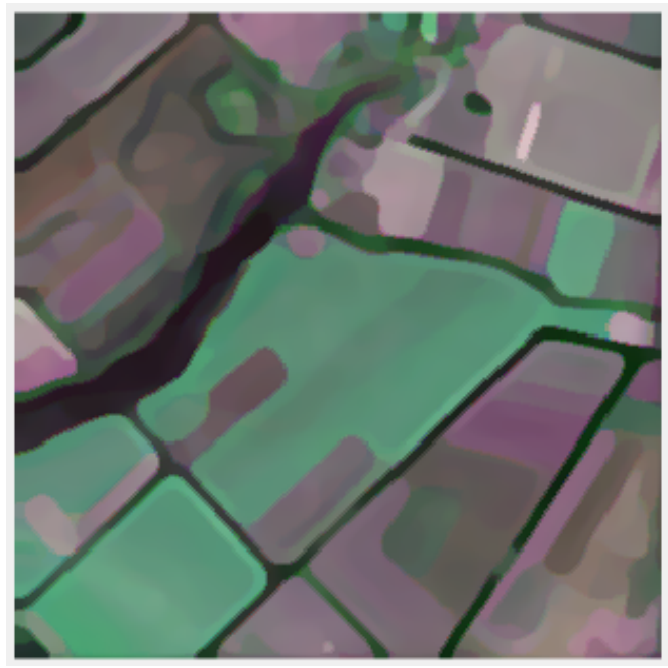

Fig. 5.7. Locally smoothed image

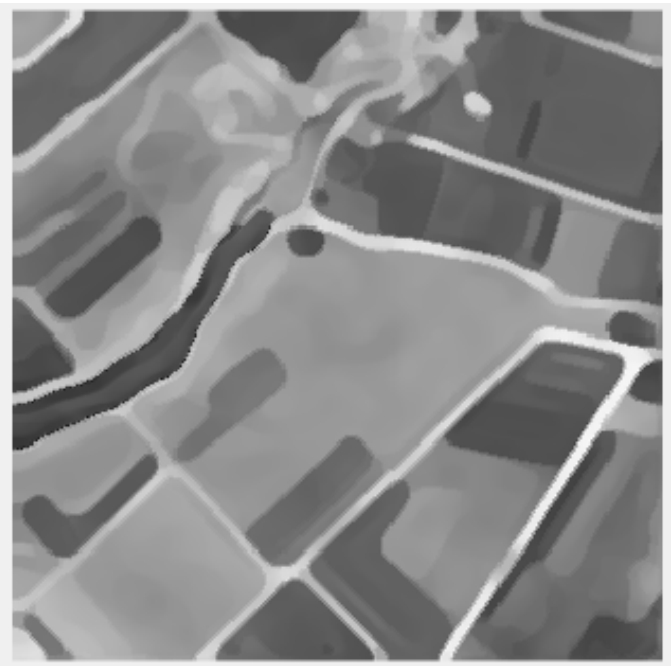

Fig. 5.8. IPVI for smoothed data

Locally smoothed image and IPVI band as a result of steady state solutions of the system $(5.5)-(5.7)$

consistency of the new statement of segmentation problem and its solvability. We derive the corresponding optimality conditions and provide their substantiation. We show that the proposed coupled optimization problem (3.7)-(3.11) is rather flexible and powerful model to the study of variational image segmentation. Mostly motivated by the crop field classification problem and the auto- 


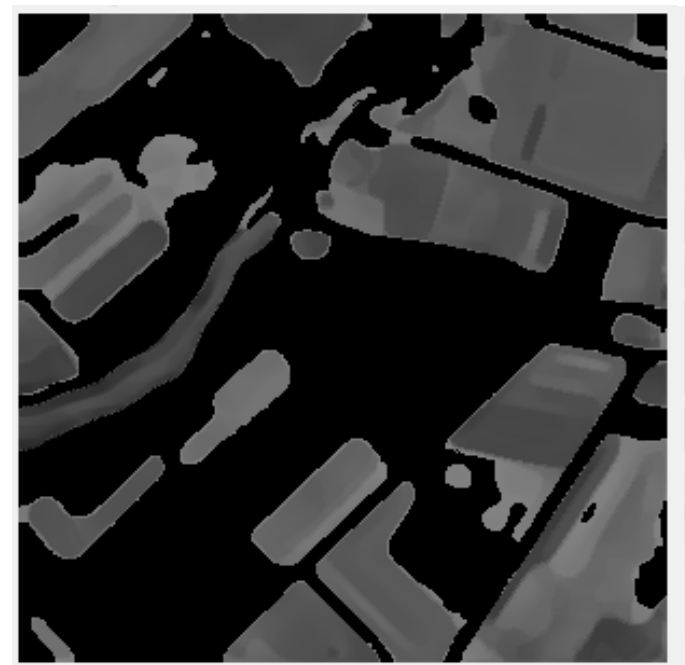

Fig. 5.9. $\Omega \backslash E^{o p t}$

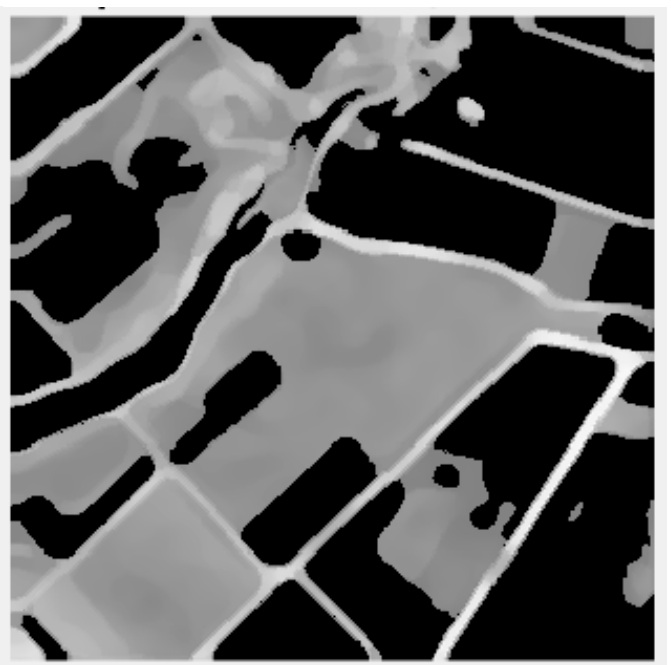

Fig. 5.10. $E^{o p t} \subset \Omega$

Result of optimal segmentation of domain $\Omega$ as a solution of piecewise constant approximation problem for the smoothed IPVI-characteristic. Here, $E$ contains a 'bright' part of $\Omega$, without black holes.

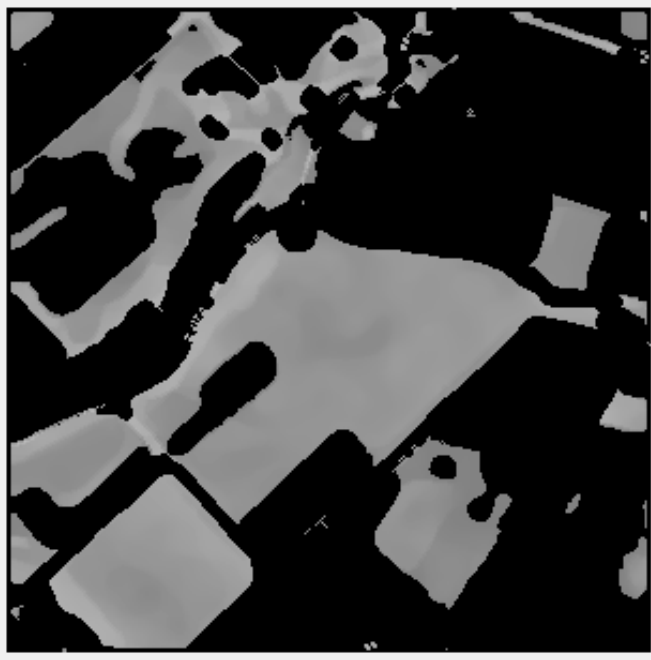

Fig. 5.11. $E^{o p t} \backslash E_{1}^{o p t}$

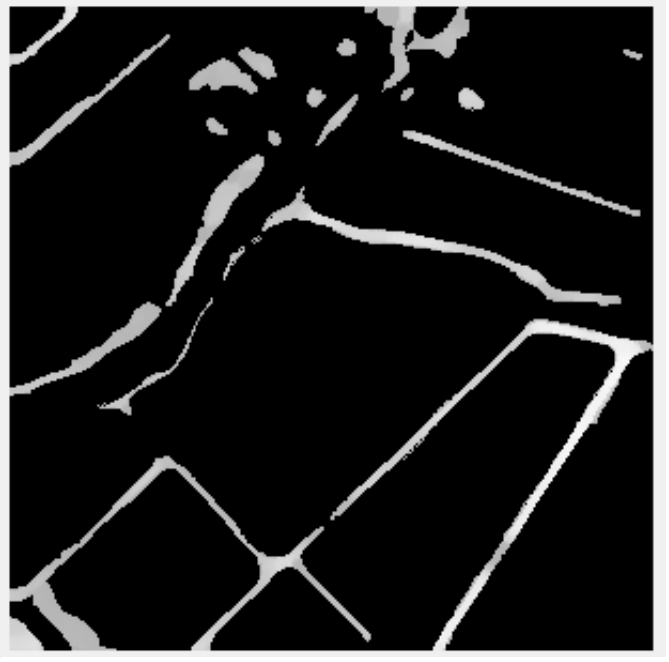

Fig. 5.12. $E_{1}^{o p t} \subset \Omega$

Result of optimal segmentation of domain $E^{o p t}$ as a solution of piecewise constant approximation problem for the smoothed IPVI-characteristic restricted to $E^{o p t}$.

mated computational methodology for the extraction of agricultural crop fields from satellite data, we provide a numerical simulation with a Sentinel-2 remote sensing image. The obtained results confirmed the effectiveness of the proposed algorithm. In particular, the experimental results indicate that IPVI-histograms 


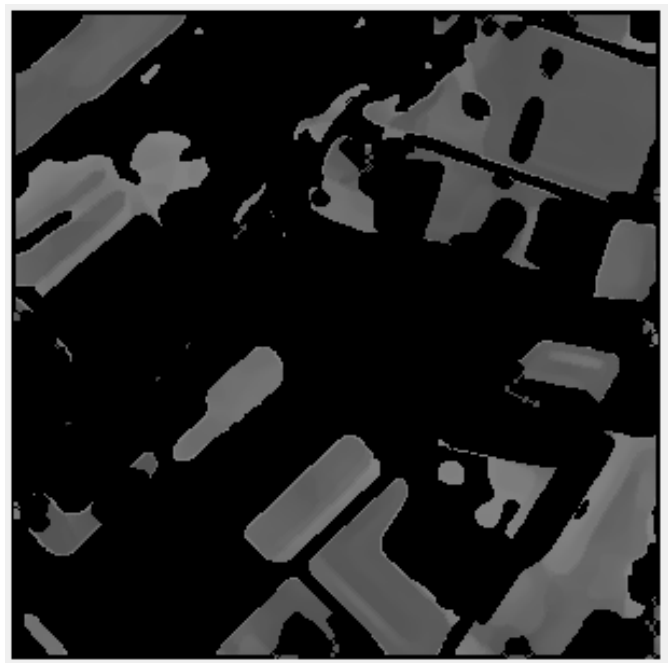

Fig. 5.13. $\left(\Omega \backslash E^{o p t}\right) \backslash E_{2}^{o p t}$

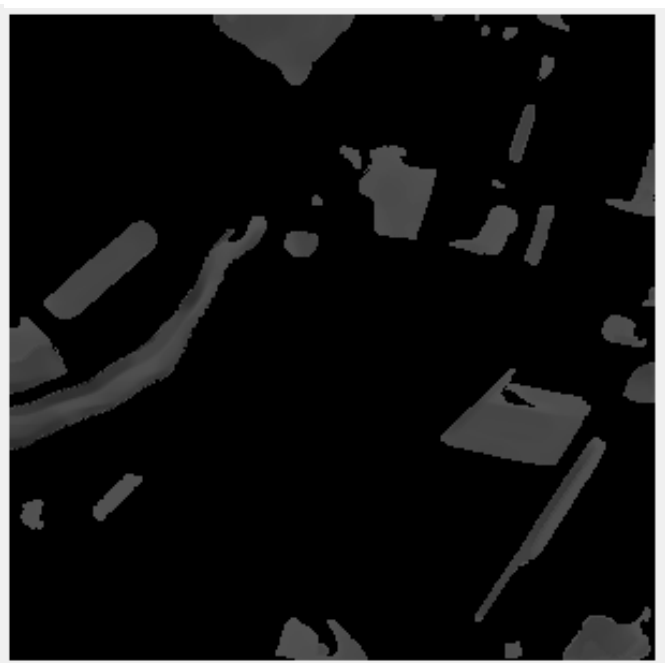

Fig. 5.14. $E_{2}^{o p t}$

Result of optimal segmentation of domain $\Omega \backslash E^{o p t}$ as a solution of piecewise constant approximation problem for the smoothed IPVI-characteristic restricted to $\Omega \backslash E^{o p t}$.

of the extracted agricultural crop fields, that have been obtained as a result optimal segmentation of the test image, have small and compactly disjointed supports in a given spectrum range. As it has been indicated in many recent publications (see $[7,12,29,31])$, this fact is crucial for the segmentation of blur and noise corrupted images with heterogeneity of their spectral and shape features. Additionally, the sensitivity analysis show that the evaluation of the smoothed versions of IPVI have a stable relationship with respect to the segmentation results.

\section{References}

1. R. ACART, C.R. Vogel, Analysis of bounded variation penalty methods for illposed problems, Inverse Problems, 10 (1994), 1217-1229.

2. L. Alvarez, P.L Lions, J.-M. Morel, Image selective smoothing and edge detection by nonlinear diffusion. II, SIAM J. Numer. Anal., 29 (3) (1992), 845-866.

3. G. Anzellotti, Pairings between measures and bounded functions and compensated compactness, Ann. Mat. Pura Appl., 135 (4) (1983), 293-360.

4. L. Ambrosio, N. Fusco, D. Pallara, Functions of bounded variation and free discontinuity problems, Oxford University Press, New York, 2000.

5. H. Attouch, G. Buttazzo, G. Michaille, Variational Analysis in Sobolev and BV Spaces: Applications to PDEs and Optimization, SIAM, Philadelphia, 2006.

6. G. Aubert, P. Kornprobst, Mathematical Problems in Image Processing: Partial Differential Equations and the Calculus of Variations, Second Edition, Series: Applied Mathematical Sciences, Vol.147, Springer, New York, 2006.

7. R. Boesch, Z. WAng, Segmentation optimization for aerial images with spacial constraints, The International Archives of the Photogrammetry, Remote Sensing and Spatial Information Sciences, XXXVII (Part B4) (2008), 285-289. 
8. D. Bucur, G. Buttazzo, Variational Methods in Shape Optimization Problems, Birkhauser, Boston, 2005.

9. R. CACCIOPpoli, Misura e integrazione sugli insieme dimensionalmente orientali I.II. Rend. Acc. Naz. Lincei, 12 (8) (1952), 3-11.

10. F. Catté, T. Coll, P.L Lions, J.-M. Morel, Image selective smoothing and edge detection by nonlinear diffusion. I, SIAM J. Numer. Anal., 29 (1) (1992), 182-193.

11. A. Chambolle, P.L. Lions, Image recovery via total variation minimization and related problems, Numer. Math., 76 (1997), 167-188.

12. Y. Chen, Q. CHEN, C. JING, Multi-resolution segmentation parameters optimization and evaluation for VHR remote sensing image based on meanNSQI and discrepancy measure, Journal of Spatial Science, https://doi.org/10.1080/14498596.2019.1615011, (2019), 1-27.

13. G. Crasta, V. De Cicco, Anzellotti's pairing theory and the Gauss-Green theorem, Advances in Mathematics, 343 (5) (2019), 935-970.

14. F. Demengel, R. Temam, Convex functions of a measure and applicatuions, Indiana Univ. Math. J., 33 (1984), 673-709.

15. I. Ekeland, R. Temam, Analyse Convexe et Problèmes Variationnels, DunodGauthier-Villars, Paris, 1974.

16. L. C. Evans, Weak convergence methods for nonlinear partial differential equations, CBMS Regional Conference Series in Mathematics, Vol.74, Published for the Conference Board of the Mathematical Sciences, Washington, DC, 1990.

17. H. FEDERER, Geometric measure theory, Springer, Berlin, 1969.

18. E. Giusti, Minimal Surfaces and Functions of Bounded Variation, Birkhäuser, Boston, 1984.

19. V.V. Hnatushenko, D.K. Mozgovyi, V.V. Vasyliev, Satellite monitoring of deforestation as a result of mining, Scientific Bulletin of National Mining University, Dnipro, 161 (5) (2017), 94-99.

20. D. Hordiuk, V. Hnatushenko, K. Maksymov, IE. Olinnyk, Semantic segmentation for ships detection from satellite imagery, 2019 IEEE 39th International Conference on Electronics and Nanotechnology (ELNANO), (2019), 454-457.

21. B. KaWohl, F. Schuricht, Dirichlet problems for the 1-Laplace operator, including the eigenvalue problem, Communications in Contemporary Mathematics, 9 (4) (2007), 515-543.

22. P.I. Kogut, G. Leugering, Optimal Control Problems for Partial Differential Equations on Reticulated Domains. Approximation and Asymptotic Analysis, Series: Systems and Control, Birkhäuser Verlag, Boston, 2011.

23. U. Massari, M. Miranda, Minimal Surfaces of Codimension One, NorthHolland, Amsterdam, 1984.

24. D. Mumford, J. Shah, Optimal approximation by piecewise smooth functions and associated variational problems, Commun. Pure. Appl. Math., 42 (5) (1989), $577-685$.

25. D.J. Mulla, Twenty five years of remote sensing in precision agriculture: key advances and remaining knowledge gaps, Biosystems Engineering, 114 (4) (2013), 358-371.

26. S. SAlsa, Partial Differential Equations in Action: From Modelling to Theory, Springer, Milan, 2008.

27. C.-B. SCHÖNLIEB, Total variation minimization with an $H^{-1}$ constraint, CRM Series 9, Singularities in Nonlinear Evolution Phenomena and Applications Proceedings, Scuola Normale Superiore Pisa, 2009, 201-232. 
28. L. VESE, A study in the BV space of a denoising-deblurring variational problem, Appl. Math. Optim., 44 (2001), 131-161.

29. P. Xiao, X. Zhang, H. Zhang, R. Hu, X. Feng, Multiscale optimized segmentation of urban green cover in high resolution remote sensing image, Remote Sensing, 10 (Article 1813) (2018), 1-20.

30. J. XuE, B. Su, Significant remote sensing Vegetation Indices: A review of developments and applications, Hindawi Journal of Sensors, 2017 (Article ID 1353691) (2017), 1-17.

31. L. YAn, D.P. Roy, Automated crop field extraction from multi-temporal Web Enabled Landsat Data, Remote Sensing of Environment, 144 (2014), 42-64.

32. YE. YuAn, C. He, Adaptive active contours without edges, Math. and Computer Modelling, 55 (2012), 1705-1721. 